\title{
Mitochondrial Regulation by PINK1-Parkin Signaling
}

\author{
Yuzuru Imai \\ Department of Neuroscience for Neurodegenerative Disorders, Juntendo University Graduate School of Medicine, \\ 2-1-1 Hongo, Bunkyo-ku, Tokyo 113-8421, Japan \\ Correspondence should be addressed to Yuzuru Imai, yzimai@juntendo.ac.jp
}

Received 9 September 2012; Accepted 30 October 2012

Academic Editors: A. Fraldi, A. Hergovich, and C. Reynaud

Copyright (c) 2012 Yuzuru Imai. This is an open access article distributed under the Creative Commons Attribution License, which permits unrestricted use, distribution, and reproduction in any medium, provided the original work is properly cited.

\begin{abstract}
Two genes responsible for the juvenile Parkinson's disease (PD), PINK1 and Parkin, have been implicated in mitochondrial quality control. The inactivation of PINK1, which encodes a mitochondrial kinase, leads to age-dependent mitochondrial degeneration in Drosophila. The phenotype is closely associated with the impairment of mitochondrial respiratory chain activity and defects in mitochondrial dynamics. Drosophila genetic studies have further revealed that PINK1 is an upstream regulator of Parkin and is involved in the mitochondrial dynamics and motility. A series of cell biological studies have given rise to a model in which the activation of PINK1 in damaged mitochondria induces the selective elimination of mitochondria in cooperation with Parkin through the ubiquitin-proteasome and autophagy machineries. Although the relevance of this pathway to PD etiology is still unclear, approaches using stem cells from patients and animal models will help to understand the significance of mitochondrial quality control by the PINK1-Parkin pathway in PD and in healthy individuals. Here I will review recent advances in our understanding of the PINK1-Parkin signaling and will discuss the roles of PINK1-Parkin signaling for mitochondrial maintenance and how the failure of this signaling leads to neurodegeneration.
\end{abstract}

\section{Introduction}

While eukaryotic cells have acquired the highly efficient power-generating system of aerobic respiration by incorporating mitochondria into the cytosol, they can suffer from problems related to uncontrollable oxidization. Nondividing cells or tissues with high energy demands in long-living animals require countermeasures against this issue because mitochondrial dysregulation has been implicated as one cause of neurodegeneration.

The neuropathology of Parkinson's disease, the second most common neurodegenerative disorder after Alzheimer's disease, is characterized by the degeneration of dopaminergic neurons in the midbrain. Mitochondrial dysfunction has long been a suspected cause of PD because the Parkinsonisminducing neurotoxin 1-methyl-4-phenyl-1,2,3,6-tetrahydropyridine (MPTP) is as a selective inhibitor of mitochondrial complex I. Reduced complex I activity has also been reported in autopsied brains and platelets from patients with sporadic PD [1-3], while mutations or polymorphisms in mitochondrial DNA are implicated in the genetic risk for
PD [4]. Animals treated with a variety of mitochondrial toxins, including MPTP, 6-hydroxy-dopamine (6-OHDA), rotenone, and paraquat, partly recapitulate $\mathrm{PD}$ pathology, suggesting that mitochondrial dysfunction and oxidative stress are key elements in PD etiology [5].

The genetic study of PD cases, including familial ones, has furthered molecular approaches to the understanding the pathogenic mechanisms underlying neurodegeneration in PD. Among the identified monogenic and susceptibility genes, Parkin, PINK1, DJ-1, and HtrA2/Omi are implicated in mitochondrial regulation, and recent molecular genetic and cell biological studies have revealed that Parkin and PINK1 play essential roles in mitochondrial quality control. In this paper, we focus on the latest studies of mitochondrial quality control by PINK1-Parkin signaling, including mitophagy for damaged mitochondria, and discuss how the loss of function of these genes may lead to neurodegeneration. The relevance and importance of mitophagy in PD is also described in detail in [6]. The progress of research on a various types of mitophagy is well reviewed in [7]. 


\section{Genetic Association between the PD Genes Parkin and PINK1}

An autosomal recessive form of juvenile PD (AR-JP) linked to the PARK2 locus, which is the major cause of juvenile PD, is caused by mutations in the Parkin gene [8]. The gene product contains a ubiquitin-like (Ubl) domain at the N-terminus and two RING fingers flanking a cysteine-rich domain, termed in between RING fingers (IBR), with ubiquitin ligase (E3) activity [9-11] (Figure 1). The RING-in-betweenRING- (RBR-) containing E3 family proteins have recently been proposed to possess a hybrid E3 activity with properties of both HECT-type and RING-finger-type E3s $[12,13]$. The Ubl domain has been shown to autoinhibit the C-terminal RBR-containing region intramolecularly [14]. Although several Parkin-deficient mouse lines have been generated; most of them did not fully recapitulate dopaminergic neurodegeneration, which hindered the determination of the pathophysiological role of the Parkin protein in vivo [15-18]. A breakthrough was achieved in genetic studies using Drosophila models for Parkin. Loss of the Parkin gene in Drosophila results in the massive degeneration of muscle tissues and a defect in spermatogenesis that is caused by mitochondrial degeneration $[19,20]$. Similar mitochondrial phenotypes were found in Drosophila lacking PINK1, which is associated with another recessive form of juvenile PD and encodes a serine-threonine kinase with a mitochondrial targeting signal at the N-terminus [21-23] (Figure 1). Genetic epistasis studies have suggested that PINK1 is upstream of Parkin and that these two genes are indispensable for the maintenance of mitochondrial functions in Drosophila [21-23].

\section{PINK1 and Parkin Are Involved in the Regulation of Mitochondrial Dynamics}

One phenotypic feature produced by the loss of PINK1 is the accumulation of elongated or aggregated mitochondria in the flight muscles and the central dopaminergic neurons, which is closely associated with mitochondrial degeneration [23-26] (Figure 2). These mitochondrial defects cause agedependent motor impairment and decreased sperm fertility. A similar morphological abnormality has been observed in Parkin-deficient flies, in which highly fused and swollen mitochondria with indistinct cristae are observed [26, 27] (Figure 2). Intriguingly, PINK1 and Parkin mutant phenotypes are partly rescued by increased Drp1 activity, which is a component of the mitochondrial fission machinery, or by the reduced activity of Mitofusin (Mfn) or optic atrophy 1 (OPA1), which are involved in mitochondrial fusion events [24-26]. Abnormal mitochondrial morphology and dynamics are also observed in mammalian cultured cells and hippocampal and dopaminergic neurons $[25,28]$. These findings suggest that PINK1 and Parkin are implicated in the regulation of mitochondrial morphology and the maintenance of mitochondrial function in both insects and mammals.

\section{Parkin Is Involved in the Elimination of Damaged Mitochondria through the Mitophagy Pathway}

Parkin is localized in the cytosol of cultured cells, but it remained unknown how Parkin regulates mitochondria. One cell biological study reported that when the mitochondrial membrane potential in cultured mammalian cells is disrupted by mitochondrial damaging reagents, such as carbonyl cyanide $m$-chlorophenylhydrazone (CCCP), Parkin is translocated to mitochondria with low membrane potential [29] (Figure 3). This translocation induces the LC3-mediated autophagic elimination of the damaged mitochondria, called mitophagy [29] (Figures 4 and 5). The mitochondrial accumulation of proteins that are poly-ubiquitinated with mainly Lys63-linked polyubiquitin and only a small portion of Lys48 linkages [30-32] recruits the ubiquitin- and LC3-binding adaptor protein p62/SQSTM1 [33-35] and ubiquitin-binding deacetylase HDAC6 [30] after Parkin translocation. Although the details of this mechanism are unresolved, Lys63-linked polyubiquitination may contribute to the proteasomal degradation of mitochondrial proteins [36] and the HDAC6- and/or p62-mediated sequestration of mitochondria [30, 31]. Depolarized mitochondria treated with CCCP or paraquat accumulate in the perinuclear compartment in a p62/SQSTM1-dependent manner [33-35], which is followed by the activation of autophagic isolation and subsequent lysosomal degradation of the mitochondria [29]. The clustering of ubiquitinated mitochondria by p62 and HDAC6 is reminiscent of the sequestration of ubiquitinated proteins, called aggresomes $[37,38]$. The class III phosphatidylinositol 3-kinase (PI3K) complex activator Ambra1 and mitochondrial protein p32 are characterized as Parkinbinding partners involved in mitophagy and mitochondrial function $[39,40]$. Ambral is recruited in a Parkin-dependent manner to perinuclear clusters of depolarized mitochondria and activates class III PI3K, which facilitates the autophagic elimination of mitochondria, a later stage of mitophagy. p32, which is primarily localized in the mitochondrial matrix, was shown to regulate the mitochondrial morphology and dynamics by decreasing the protein level of Parkin although the detailed molecular mechanism of p32 remains unclear [40]. p32 is suggested to support oxidative phosphorylation (OXPHOS) through the promotion of protein synthesis for mitochondrial respiratory complexes [41].

How is the autophagic machinery targeted to mitochondria? In yeast, the outer mitochondrial protein ATG32 is reported to recruit the autophagic machinery $[44,45]$. Although there are no ATG32 homologs in higher animals, BNIP3 (BCL2 and adenovirus E1B $19 \mathrm{kDa}$-interacting protein 3) and NIX/BNIP3 like (BNIP3 L), which belong to the $\mathrm{BH} 3$-only mitochondrial protein family, induce both cell death [46-48] and mitophagy [49-51]. NIX is involved in programmed mitochondrial clearance by mitophagy during reticulocyte maturation $[49,52]$ and is reported to be required for Parkin translocation to depolarized mitochondria treated with CCCP [51]. However, it is unclear whether NIX is a key regulator of the PINK1-Parkin 

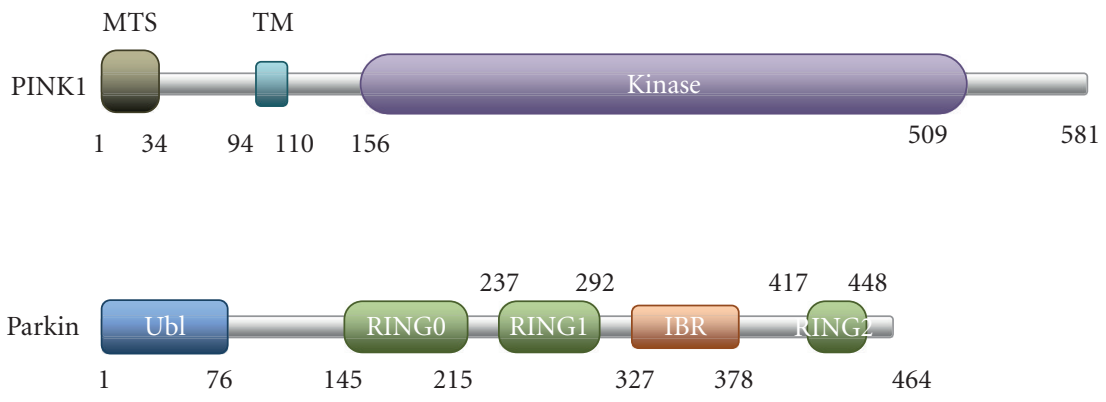

FIGURe 1: PINK1 and Parkin proteins. MTS, mitochondrial targeting sequence; TM, transmembrane domain; Ubl, ubiquitin-like domain; RING, Ring finger motif; IBR, in between RING fingers domain. RING0 has been characterized as a new domain with a similarity to conventional RING1 and RING2 domains [42]. The numbers correspond to the residue numbers for boundaries of the indicated domains.

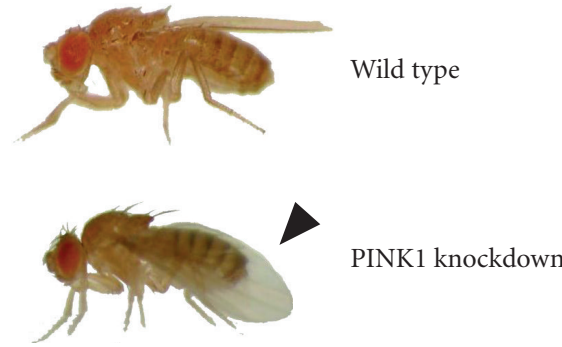

(a)
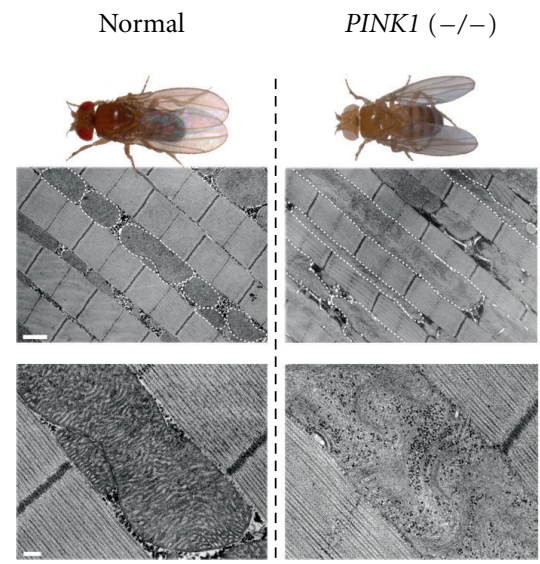

(b)

FIGURE 2: PINK1 and Parkin are required for the mitochondrial maintenance in Drosophila melanogaster. (a) Wings are kept in a horizontal position in wild-type fly (upper) while PINK1 knockdown in the muscles results in a drooped wing posture (arrowhead) due to the degeneration of the flight muscle mitochondria (lower). Data were adapted from [43]. (b) Transmission electron microscopy (TEM) analysis of the indirect flight muscle and morphology of mitochondria in 2-day-old adult flies. PINK1- and Parkin-deficient flies showed elongated and swollen mitochondria with indistinct cristae. Some mitochondria are outlined with broken lines to highlight morphology. Overhead view of wild-type (normal), PINK1- and Parkin-deficient flies is also shown. Scale bars $=1 \mu \mathrm{m}$ in upper panels and $200 \mathrm{~nm}$ in lower. Original data were published in [27].

pathway because NIX-like proteins have not been found in Drosophila. In contrast, Nix has been characterized as a key mediator of a new type of mitochondrial quality control system, designated MALM (Mieap-induced accumulation of lysosome-like organelles within mitochondria) $[53,54]$. MALM is involved in the selective degradation of oxidized mitochondrial proteins, which is performed by lysosome-like organelles independent of the PINK1-Parkin pathway.

\section{PINK1 Regulates the Mitochondrial Translocation of Parkin}

The translocation of Parkin from the cytosol to the mitochondria, which requires intact PINK1 with kinase activity, is an initial step of the mitophagy process in mammalian [33, 55-58] and Drosophila cultured cells [59] (Figure 6). Most of the pathogenic mutations found in PINK1 and Parkin compromise the mitochondrial translocation activity of Parkin, which was partly confirmed using a neuronal culture derived from iPS cells of PINK1-linked PD cases [60]. Thus, Parkinmediated mitophagy may be closely associated with the etiology of juvenile PD caused by PINK1 and Parkin mutations.

The E3 activity of Parkin is activated through an unknown mechanism after mitochondrial translocation, and it degrades several proteins localized in the mitochondrial outer membrane through the ubiquitin-proteasome pathway, which includes Mfn1 and Mfn2 [32, 61, 62], Drp1 [63], voltage-dependent anion channel 1 (VDAC1) [32, 33], and Bcl-2 [64]. The degradation of the mitochondrial elongation factor Mfn by Parkin, also observed in Drosophila cultured cells, contributes to the fragmentation of the mitochondria during mitophagy $[59,65]$. This finding is consistent with observations in Drosophila, in which the loss of PINK1 or Parkin leads to mitochondrial elongation, and a reduction of Mfn activity partly rescues the mitochondrial degeneration [24-26]. However, the elimination of Mfn by Parkin and the perinuclear aggregation of mitochondria by 

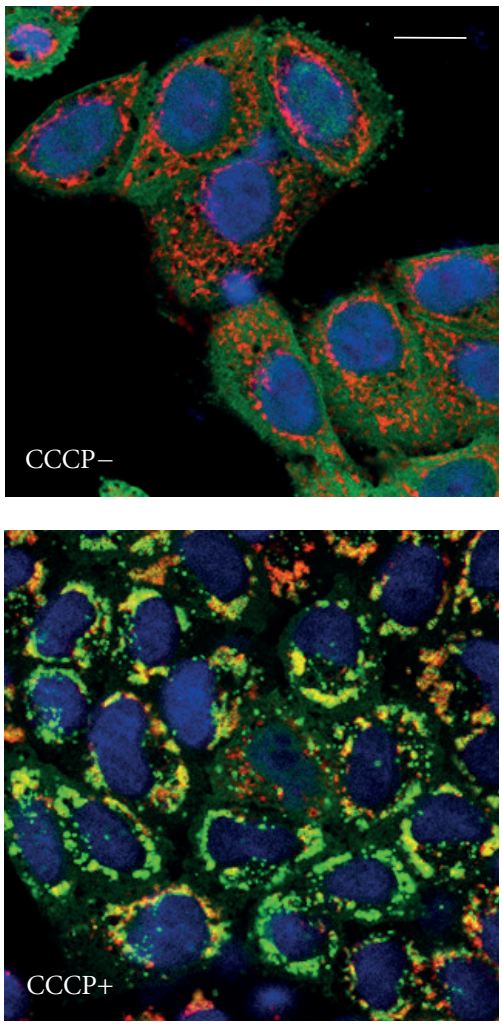

Figure 3: Mitochondrial translocation of Parkin. HeLa cells expressing GFP-tagged Parkin (green) were treated with $(+)$ or without (-) $10 \mu \mathrm{M}$ CCCP for 3 hrs to disrupt the mitochondrial membrane potential $(\Delta \psi \mathrm{m})$. Parkin was moved to mitochondria upon the reduction of $\Delta \psi \mathrm{m}$ (stained with mitochondrial outer membrane protein Tom 20 (red), counterstained with DAPI (blue) for the nucleus). Scale bar $=10 \mu \mathrm{m}$. Original data were provided by Shiba-Fukushima et al.

p62/SQSTM1 per se appear to be dispensable for mitophagy in mammalian cells $[32,34,35]$, although the requirement of p62 is controversial [33]. As it is very likely that a p62-related protein NBR1 is also recruited to the damaged mitochondria in cultured mammalian cells, NBR1 may compensate the p62 function [32]. Mfn degradation and mitochondrial perinuclear clustering may prevent the refusion of damaged mitochondria with healthy mitochondria and the axonal transportation of damaged mitochondria [34, 61]. In addition, mitochondrial reorganization by the inactivation of $\mathrm{Mfn}$ and the recruitment of $\mathrm{p} 62$ may facilitate the isolation of mitochondria by the autophagosome [61].

\section{Molecular Regulation of PINK1 and Parkin}

PINK1 was originally isolated as one of the genes induced by the tumor suppressor PTEN, suggesting that the PI3K/AKT pathway negatively regulates PINK1 expression [66]. Later, FoxO, a downstream component of the PI3K/AKT pathway, was shown to transactivate the mammalian PINK1 promoter [67]. Although PINK1 transcripts are abundantly expressed in cultured cells and several mitochondria-rich tissues, including the heart, skeletal muscle, and testis [66], it is difficult to detect endogenous PINK1 protein, which has made it challenging to elucidate the molecular action of PINK1. However, PINK1 turned out to be accumulated in depolarized mitochondria rapidly $[55,57,58]$, suggesting that PINK1 is subjected to a posttranslational degradation (Figure 7). Several studies report that the rhomboid family protease presenilin-associated rhomboid-like protein (PARL), which is localized in the mitochondrial inner membrane (IM), processes PINK1 in a mitochondrial membrane potential-dependent manner [68-72]. Newly synthesized PINK1 in the cytosol is inserted into the IM, through the mitochondrial import activity of the Tom complex [73], and is cleaved in its putative transmembrane domain by PARL to generate the $52 \mathrm{kDa}$ form of PINK1. The $52 \mathrm{kDa}$ form is rapidly removed by a proteasome-dependent pathway, most likely after its release from the mitochondrial intermembrane space (IMS) to the cytosol [69-71].

Upon the depolarization of the mitochondrial membrane potential, IM insertion and the subsequent processing of PINK1 by PARL may be inhibited, leading to the accumulation of full-length PINK1 in the mitochondrial outer membrane $(\mathrm{OM})$, most likely facing the cytosol $[70,71,74]$. However, it remains controversial whether the processing of PINK1 by PARL is required for Parkin recruitment upon depolarization of the mitochondria, and further studies will be necessary to completely resolve the topology changes of the processed forms of PINK1 [70, 72].

The accumulation and autophosphorylation of PINK1 likely stimulate its kinase activity, which is required for the mitochondrial translocation of Parkin [73, 75]. Although the detailed molecular mechanism through which PINK1 recruits Parkin to mitochondria remains unclear, our data suggest that the PINK1-dependent phosphorylation of Parkin in the Ubl domain is involved [76]. The phosphorylation of the Parkin Ubl domain by PINK1 may also contribute to the stimulation of Parkin E3 activity through the release of the RBR region from the autoinhibitory mechanism by the Ubl domain [77].

Parkin is also regulated at the transcriptional level. The unfolded protein response (UPR) transactivates Parkin transcripts $[9,78-80]$ through ATF4, a transcription factor that is involved in the unfolded protein response (UPR) [81]. Mitochondrial damage may induce the activation of the UPR $[81,82]$ and oxidative stress [83], leading to the upregulation of Parkin expression. In other stress contexts, such as $\gamma$ irradiation or hydrogen peroxide, p53 transactivates Parkin [84]. Conversely, Parkin is reported to suppress the transcription of p53 through the direct binding of Parkin to the $p 53$ promoter region, suggesting a negative feedback mechanism [85]. The p53-dependent induction of Parkin is implicated in the regulation of glucose metabolism because the loss of Parkin activates glycolysis and reduces mitochondrial respiration, leading to the Warburg effect [84]. This phenotype seems to be associated with the accumulating evidence from tumor cell lines and model animals that Parkin functions as a tumor suppressor. Parkin gene expression is downregulated or absent in ovarian [86-88] and breast tumors [89], acute lymphoblastic leukemia and chronic myeloid leukemia [90], 

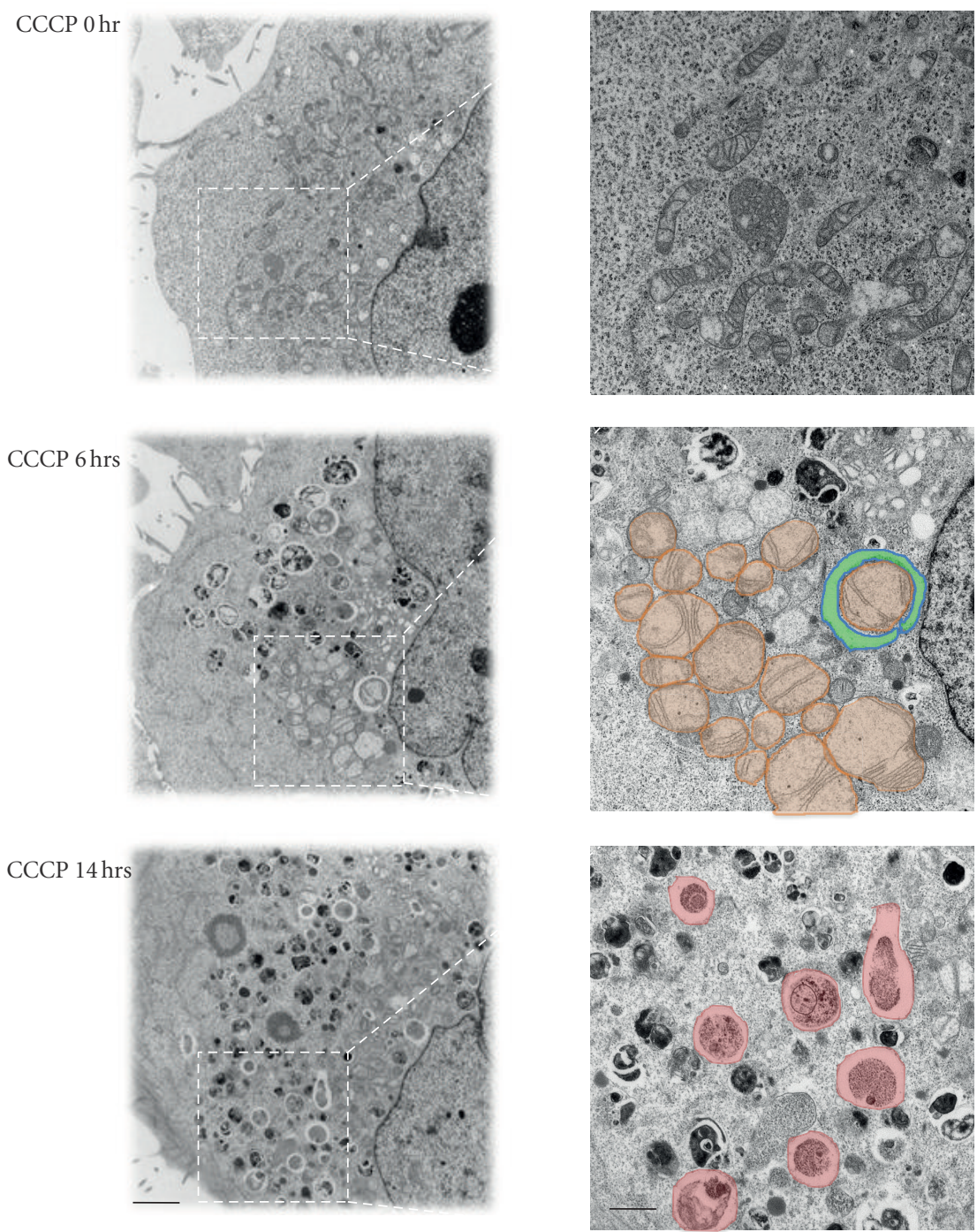

FIgURE 4: Ultramicroscopic analysis of mitophagy by Parkin. HeLa cells overexpressing Parkin were treated with CCCP for the indicated periods of time. Tubular mitochondria were seen in the cytoplasm before CCCP treatment. Swollen mitochondria (marked in orange) were observed in the perinuclear region of cytoplasm $6 \mathrm{hrs}$ after CCCP treatment. The isolation membrane (green) encircling a swollen mitochondrion was also seen. Partially digested mitochondria in the lysosome-like vacuoles (red) were observed $14 \mathrm{hrs}$ after CCCP treatment. Scale bars $=2 \mu \mathrm{m}$ (left) and $500 \mathrm{~nm}$ (right). Original data were provided by Shiba-Fukushima et al.

colorectal cancers [91], non-small-cell lung cancer [92], glioblastoma [93], and tumor cell lines [94]. The inactivation of Parkin also leads to enhanced hepatocyte proliferation and the development of hepatic tumors with characteristics of hepatocellular carcinoma in mice [95] and humans [96].

\section{PINK1 and Parkin Regulate Mitochondrial Motility}

A variety of mitochondrial proteins are ubiquitinated and degraded through the ubiquitin-proteasome pathway after the mitochondrial translocation of Parkin [32]. It is not clear whether all of these proteins are ubiquitinated by Parkin. Among the mitochondrial proteins that are degraded, Mfn (see above) and Miro are well characterized as Parkin substrates. Miro family proteins contain two GTPase domains separated by a linker region containing putative calcium-binding EF hand motifs [97]. Miro is involved in microtubule-dependent mitochondrial transport through its binding partners Milton and kinesin heavy chain (KHC) [98-100]. Miro has been identified as one of the binding partners of PINK1 [101] and was isolated as a gene that modulates the phenotypes of PINK1 mutant flies [102]. Two recent reports have demonstrated that Miro is degraded through the PINK1-Parkin pathway, which aids in the removal of damaged mitochondria from mitochondrial trafficking pathways [102, 103] (Figure 8). Wang et al. have also shown that the phosphorylation of Miro by PINK1 is a prerequisite for its degradation via Parkin [103], while another study failed to observe Miro phosphorylation or the requirement of this modification for Miro degradation [102]. 


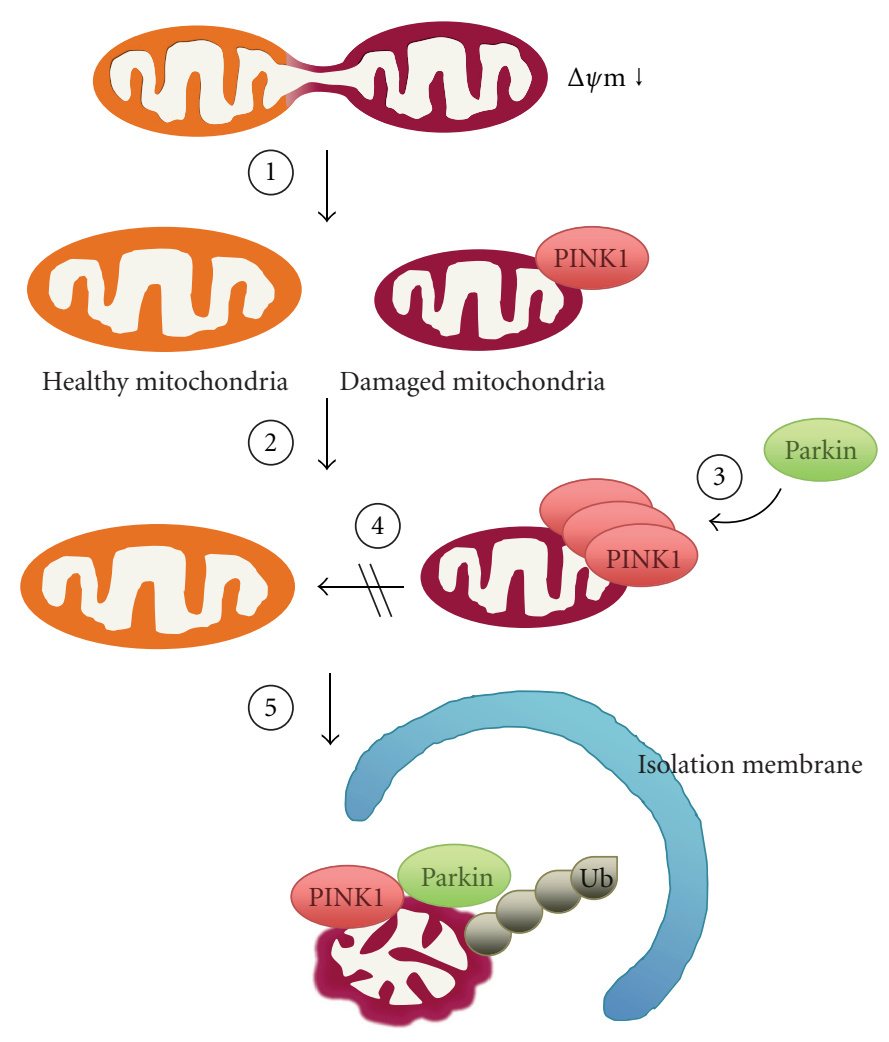

FIGURE 5: Mitophagy mediated by PINK1 and Parkin. Fusion/fission is required for the maintenance of a healthy mitochondrial population. Mitochondrial fusion is thought to require the exchange of a set of internal components, including copies of the mitochondrial genome, respiratory proteins, and metabolic products. Mitochondrial fission may play a role in the removal of damaged mitochondria $(1,2)$ with a reduced $\Delta \psi \mathrm{m}$ through an autophagy-lysosomal pathway, that is, "mitophagy." PINK1 is constitutively degraded in healthy mitochondria (see also Figure 3). Upon a decrease in $\Delta \psi \mathrm{m}$, PINK1 is stabilized in the mitochondrial outer membrane (OM) (3). The accumulation of PINK1 induces the translocation of Parkin from the cytosol to the mitochondria (3), which leads to Parkin-dependent mitochondrial protein degradation, through which Mfn is degraded to prevent healthy mitochondria from refusing with damaged mitochondria (4). Parkin subsequently activates the autophagy machinery, which includes induction of the isolation membrane for autophagy (5).

\section{Possible Involvement of DJ-1 and HtrA2 in the PINK1-Parkin Pathway}

DJ-1, which exerts neuroprotective activities by scavenging hydrogen peroxide through self-oxidation, has been reported to be involved in mitochondrial maintenance [104-109]. Recently accumulated data suggest that DJ-1 functions in parallel to the PINK1-Parkin pathway to control mitochondrial activity in cultured cells $[110,111]$ and Drosophila $[110,112]$, while DJ-1/PINK1/Parkin triple knock-out mice exhibit no degenerative phenotypes in the nigrostriatal system [113]. In the context of mitophagy, however, reactive oxygen species (ROS) sensitize the mitochondrial translocation of Parkin in primary cultured neurons and other cells, and this sensitization is enhanced by the loss of DJ-1 [114]. A related finding has been reported that increased glutathione S-transferase activity protects dopaminergic neurons in Parkin-deficient flies, suggesting that ROS overproduction is an important component of the pathology of PINK1-Parkin defects [115].

Although the association between PD and the HtrA2 gene, which encodes a mitochondrial serine protease and is involved in mitochondrion-dependent apoptosis [116119], is disputed [120-122], the loss of the HtrA2 gene leads to the selective loss of striatal neurons in mice, causing a Parkinsonian neurodegenerative phenotype [123]. It has been suggested that PINK1-dependent phosphorylation of HtrA2 enhances the protease activity of HtrA2, which is required for stress resistance [124]. In contrast, it has been shown that HtrA2 cleaves and inactivates Parkin under stress condition [125]. Genetic studies in Drosophila [126] and mice [127] do not suggest that HtrA2 is implicated in the PINK1-Parkin pathway.

\section{Dysregulation of Mitochondrial Control and Possible Disease Relevance}

Although PINK1-Parkin signaling appears to be conserved, at least between mammals and Drosophila, PINK1- and Parkin-deficient mice do not recapitulate the symptoms of PD. The molecular mechanism underlying mitochondrial quality control by the PINK1-Parkin pathway has been mostly characterized in cultured cells using mitochondriadamaging reagents. Therefore, the relevance of mitophagy 

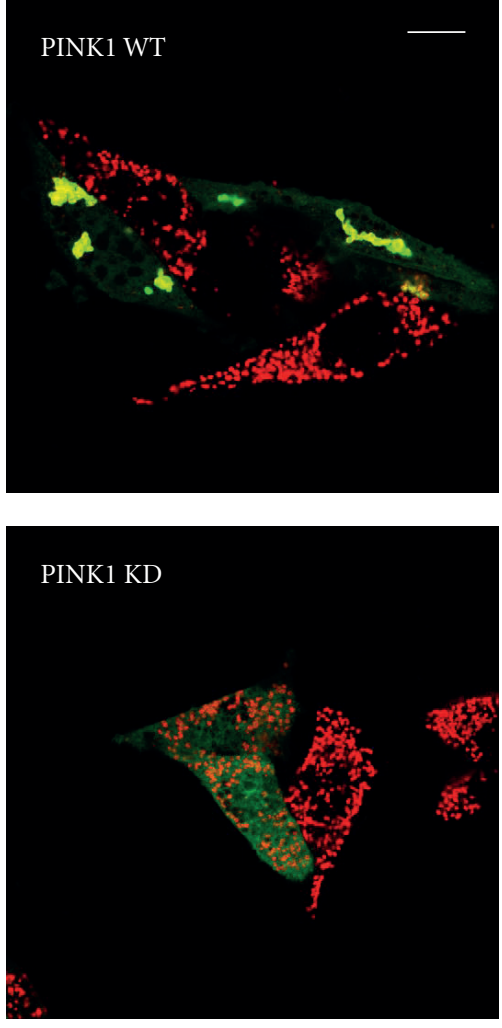

FIGURE 6: PINK1 kinase activity is required for the mitochondrial translocation of Parkin. Wild-type (WT) or kinase-dead (KD) PINK1 together with GFP-Parkin (green) was introduced in mouse embryonic fibroblasts derived from a PINK1-deficient mouse. GFPParkin was located on the mitochondria (red; Tom 20) in cells expressing PINK1 WT but not PINK1 KD after CCCP treatment. Scale bar $=10 \mu \mathrm{m}$. Original data were provided by Shiba-Fukushima et al.

observed in the cultured cells to PD etiology remains debatable. However, the accumulating evidence of mitochondrial abnormalities in animal models and PD patients has increased our understanding of the pathogenesis of the disease. It has been shown that mitochondrial populations harboring mutant mitochondrial genomes are selectively eliminated by Parkin [128]. The ubiquitination and elimination of $\mathrm{Mfn}$ do not occur in human fibroblasts derived from PINK1- and Parkin-linked PD patients following oxidative stress induction and depolarization treatment of the mitochondria [129]. Recent studies on Parkin- and PINK1-deficient mice have reported morphological and functional alterations of the mitochondria in neurons [130, 131] and astrocytes [132]. Like the muscle degeneration in Drosophila, the function of the heart, which depends on highly developed cardiac muscle mitochondria, is also impaired with increased oxidative stress in PINK1 null mice [133]. Ischemic preconditioning has cardioprotective effects in heart failure models in which the mitochondrial translocation of Parkin is induced [134]. The deletion of Parkin in mice abolishes this effect [134]. Although the role of PINK1 and Parkin in the cardiac function of humans is yet unknown, it is worth noting that the prevalence of heart failure in elderly PD patients is twice that of elderly individuals without PD [135].

Interestingly, a missense mutation of the mitophagyassociated PARL gene found in PD cases abolishes PINK1 processing activity and subsequent Parkin-mediated mitophagy [72]. No polymorphisms in the Miro gene have been linked to PD to date [136], while the inactivation of Mfn2 but not Mfn1 in dopaminergic neurons of the mouse brain resulted in progressive degeneration of the nigrostriatal pathway, suggesting a functional difference between Mfn1 and Mfn2 in neurons [137, 138]. Mutations in Mfn2 cause the Charcot-Marie-Tooth disease type $2 \mathrm{~A}$, a peripheral nerve disorder characterized by a slowly progressive degeneration of the muscles of the extremities [139-141]. Mfn2 has been shown to be required for axonal mitochondrial transport by direct binding to Miro [142]. In this context, the mitochondrial fusion activity is not suggested to be essential for the mitochondrial transport because knockdown of OPA1, another protein for mitochondrial fusion, does not affect the mitochondrial motility [142].

\section{Therapeutic Approach to the Mitochondrial Defects Caused by the Dysregulation of PINK1-Parkin Signaling}

The impairment of mitochondrial complex I activity has been implicated in PINK1- and Parkin-linked PD cases and animal models [131, 143-148]. The addition of complex I and II substrates has been shown to improve the reduced respiration activity caused by inactivated PINK1 in cultured neurons [148]. The introduction of the yeast NADH dehydrogenase gene, which compensates for complex I activity, effectively rescues the PINK1 mutant phenotype in Drosophila but does not ameliorate the Parkin phenotype, suggesting that the complex I regulation pathway is downstream of or parallel to PINK1 but upstream of Parkin [149].

A recent genetic screen using a Drosophila PINK1 model isolated an enzyme producing vitamin $\mathrm{K}_{2}$ as a strong modifier gene and revealed that vitamin $\mathrm{K}_{2}$ functions as a mitochondrial electron carrier [150]. Because vitamin $\mathrm{K}_{2}$ administration improves both PINK1 and Parkin phenotypes in Drosophila, vitamin $\mathrm{K}_{2}$ may be a promising therapy for general PD and PINK1- or Parkin-linked PD. The suppression of the mammalian target of rapamycin complex 1 (mTORC1) signaling for general protein translation [151-153] and stimulation of mitochondrial biogenesis $[154,155]$ may also be beneficial for the prevention of PD pathogenesis (Figure 9).

\section{Conclusions}

One of the neuropathological features of PD is the accumulation of protein inclusions called Lewy bodies, which implies a dysfunction of protein quality control regulated by the ubiquitin-proteasome and autophagy pathways. Indeed, some PD and PD-related genes are characterized as components of the protein quality control system (e.g., LRRK2 [157-162], ATP13A2 [163-166], HtrA2 [167, 168], and 


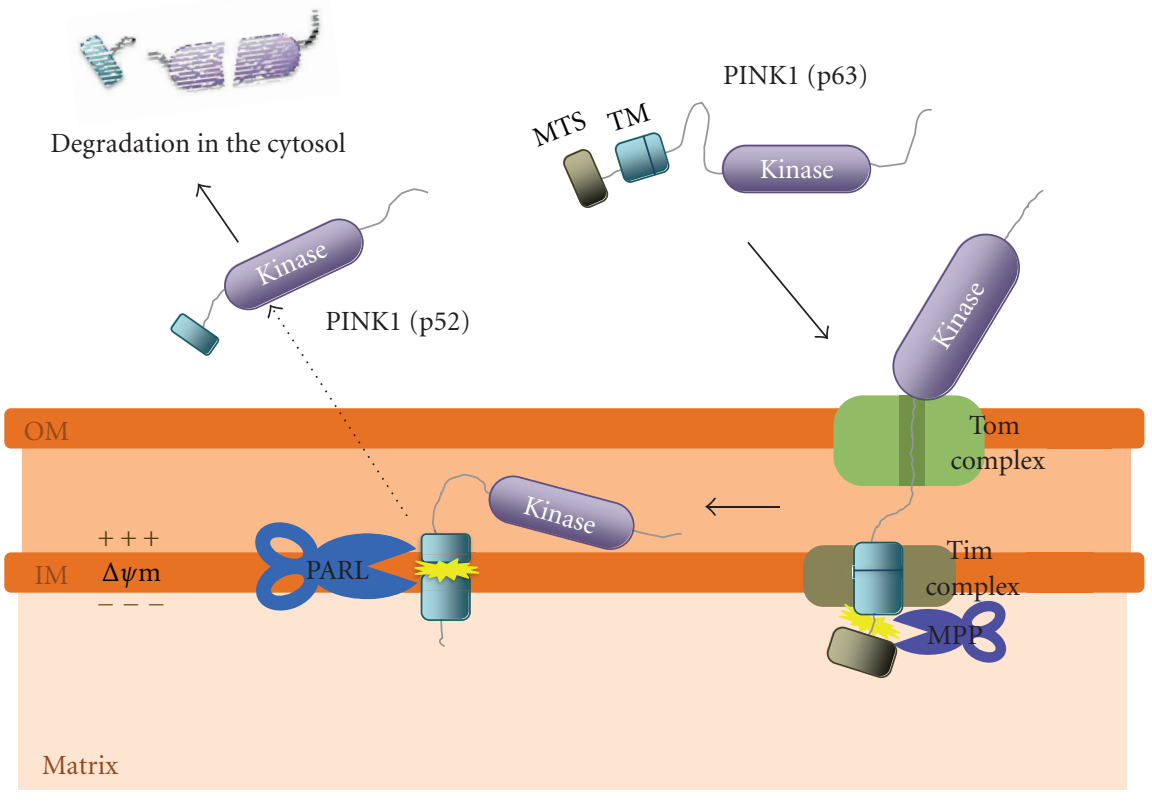

Healthy mitochondria

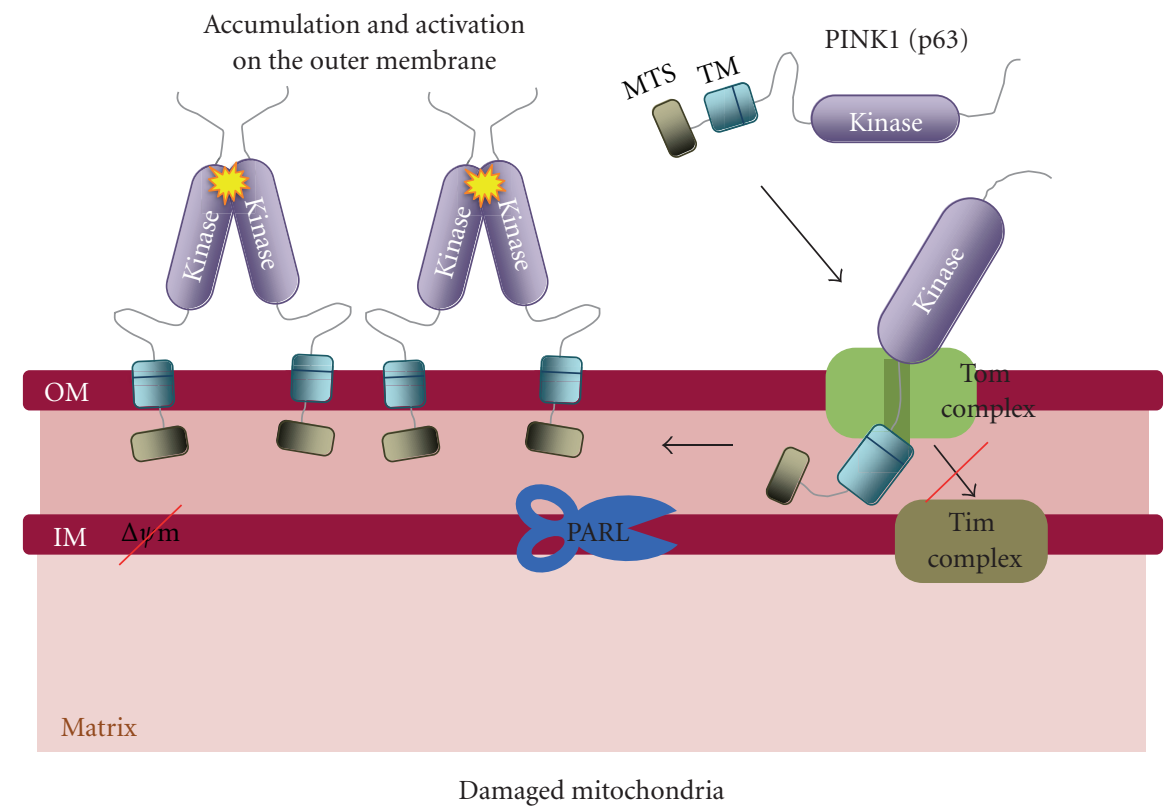

FIGURE 7: Proposed model of posttranslational processing of PINK1. Upper: newly synthesized PINK1 (p63) is targeted to the mitochondrial inner membrane (IM) via the Tom and Tim complexes. Full-length PINK1 is processed by mitochondrial processing protease (MPP), which cleaves the mitochondrial targeting sequence to generate the $60 \mathrm{kD}$ form of PINK1 [130, 156]. PINK1 is then cleaved to a $52 \mathrm{kD}$ form within the IM by PARL $[68-71,156]$. The $52 \mathrm{kD}$ PINK1 is released from the cytosol and degraded through proteasome activity. Lower: upon a decrease in $\Delta \psi \mathrm{m}$, PINK1 is accumulated at the OM, most likely due to the inhibition of its transportation to the Tim complex.

FBXO7 $[169,170])$ or abnormal proteins themselves $(\alpha-$ Synuclein [171-173], Tau [174-180]). In fission yeast, the impairment of the ubiquitin-proteasome pathway induces the accumulation of reactive oxygen species in mitochondria [181]. These mitochondria are removed from the autophagy pathway [181]. In a Drosophila model, it has been shown that mitochondria with accumulated misfolded proteins are also removed by Parkin [182]. The observation evokes endoplasmic-reticulum- (ER-) associated protein degradation (ERAD), which eliminates misfolded or unassembled proteins from the ER [183]. Intriguingly, a AAA+ ATPase p97/VCP, which is required for ERAD along with proteasome 


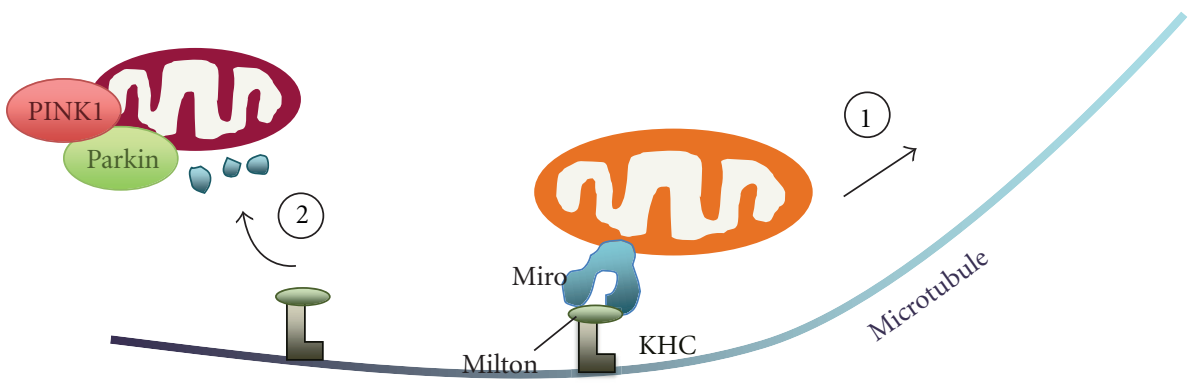

FIgURE 8: Quarantine control of mitochondrial transportation by PINK1-Parkin signaling. Mitochondria are transported along the microtubule network through the Miro-Milton-KHC complex (1). In neurons, the transportation of healthy mitochondria to the nerve terminal ensures a stable energy supply for a variety of synaptic activities. PINK1 is activated in mitochondria with reduced $\Delta \psi \mathrm{m}$ and recruits Parkin, which results in Miro degradation; thus, damaged mitochondria are selectively removed from the mitochondrial transport machinery (2). These mitochondria may be further eliminated by PINK1-Parkin-mediated mitophagy.

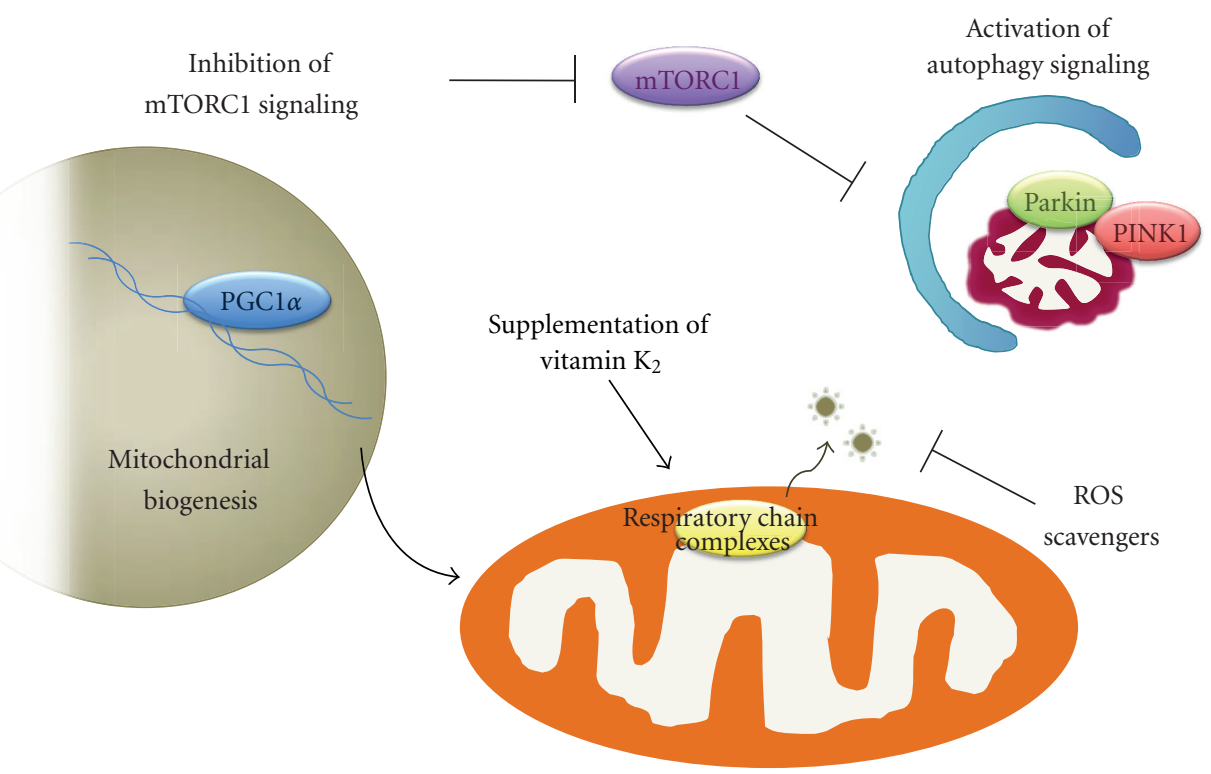

FIGURE 9: Signal pathways that modulate the PINK1-Parkin pathway. Inhibition of the mTORC1 pathway stimulates mitochondrial degeneration in Drosophila, while supplementation with vitamin $\mathrm{K}_{2}$ or ROS scavengers ameliorates defects in respiratory chain activity. Increased mitochondrial biogenesis by the activation of peroxisome proliferator-activated receptor- $\gamma$ coactivator- (PGC)- $1 \alpha$ and autophagy machinery may improve mitochondrial activity.

activity, is involved in the elimination of proteins on the OM during mitophagy [61]. Thus, the pathways for protein quality control and mitochondrial maintenance are likely to be linked to each other.

Two gene products implicated in juvenile PD, PINK1 and Parkin, have recently been implicated in mitochondrial quality control. PINK1-Parkin-mediated mitophagy includes two major components: commitment to elimination by PINK1 and Parkin and execution of elimination by autophagy. While canonical autophagy components appear to be employed in the autophagy stage, the molecular mechanism through which PINK1 and Parkin signal the commitment to elimination remains largely unclear [29, $57,152,184]$. This is obviously an important issue, and its resolution might lead to the identification of new genes associated with neurodegenerative disorders and new targets for a therapeutic approach to PD.

\section{Acknowledgments}

The author thanks K. Shiba and T. Sawada for providing data. This research is supported by the Novartis Foundation Research Grant, the Naito Foundation Research Grant, the Ichiro Kanehara Foundation Research Grant, and a Grantin-Aid for Young Scientists from MEXT in Japan.

\section{References}

[1] W. D. Parker Jr., S. J. Boyson, and J. K. Parks, "Abnormalities of the electron transport chain in idiopathic Parkinson's disease," Annals of Neurology, vol. 26, no. 6, pp. 719-723, 1989.

[2] A. H. V. Schapira, J. M. Cooper, D. Dexter, J. B. Clark, P. Jenner, and C. D. Marsden, "Mitochondrial complex I deficiency in Parkinson's disease," Journal of Neurochemistry, vol. 54, no. 3, pp. 823-827, 1990. 
[3] P. M. Keeney, J. Xie, R. A. Capaldi, and J. P. Bennett, "Parkinson's disease brain mitochondrial complex I has oxidatively damaged subunits and is functionally impaired and misassembled," Journal of Neuroscience, vol. 26, no. 19, pp. 5256 5264, 2006.

[4] A. Bender, K. J. Krishnan, C. M. Morris et al., "High levels of mitochondrial DNA deletions in substantia nigra neurons in aging and Parkinson disease," Nature Genetics, vol. 38, no. 5, pp. 515-517, 2006.

[5] S. Hisahara and S. Shimohama, "Toxin-induced and genetic animal models of Parkinson's disease," Parkinson's Disease, vol. 2011, Article ID 951709, 14 pages, 2011.

[6] R. L. de Vries and S. Przedborski, "Mitophagy and Parkinson's disease: be eaten to stay healthy," Molecular and Cellular Neuroscience. In press.

[7] G. Ashrafi and T. L. Schwarz, "The pathways of mitophagy for quality control and clearance of mitochondria," Cell Death and Differentiation. In press.

[8] T. Kitada, S. Asakawa, N. Hattori et al., "Mutations in the parkin gene cause autosomal recessive juvenile parkinsonism," Nature, vol. 392, no. 6676, pp. 605-608, 1998.

[9] Y. Imai, M. Soda, and R. Takahashi, "Parkin suppresses unfolded protein stress-induced cell death through its E3 ubiquitin-protein ligase activity," The Journal of Biological Chemistry, vol. 275, no. 46, pp. 35661-35664, 2000.

[10] H. Shimura, N. Hattori, S. I. Kubo et al., "Familial Parkinson disease gene product, parkin, is a ubiquitin-protein ligase," Nature Genetics, vol. 25, no. 3, pp. 302-305, 2000.

[11] Y. Zhang, J. Gao, K. K. K. Chung, H. Huang, V. L. Dawson, and T. M. Dawson, "Parkin functions as an E2-dependent ubiquitin-protein ligase and promotes the degradation of the synaptic vesicle-associated protein, CDCrel-1," Proceedings of the National Academy of Sciences of the United States of America, vol. 97, no. 24, pp. 13354-13359, 2000.

[12] D. M. Wenzel, A. Lissounov, P. S. Brzovic, and R. E. Klevit, "UBCH7 reactivity profile reveals parkin and HHARI to be RING/HECT hybrids," Nature, vol. 474, no. 7349, pp. 105108, 2011.

[13] B. Stieglitz, A. C. Morris-Davies, M. G. Koliopoulos, E. Christodoulou, and K. Rittinger, "LUBAC synthesizes linear ubiquitin chains via a thioester intermediate," EMBO Reports, vol. 13, no. 9, pp. 840-846, 2012.

[14] V. K. Chaugule, L. Burchell, K. R. Barber et al., "Autoregulation of Parkin activity through its ubiquitin-like domain," EMBO Journal, vol. 30, no. 14, pp. 2853-2867, 2011.

[15] M. S. Goldberg, S. M. Fleming, J. J. Palacino et al., "Parkindeficient mice exhibit nigrostriatal deficits but not loss of dopaminergic neurons," The Journal of Biological Chemistry, vol. 278, no. 44, pp. 43628-43635, 2003.

[16] J. M. Itier, P. Ibáñez, M. A. Mena et al., "Parkin gene inactivation alters behaviour and dopamine neurotransmission in the mouse," Human Molecular Genetics, vol. 12, no. 18, pp. 2277-2291, 2003.

[17] R. Von Coelln, B. Thomas, J. M. Savitt et al., "Loss of locus coeruleus neurons and reduced startle in parkin null mice," Proceedings of the National Academy of Sciences of the United States of America, vol. 101, no. 29, pp. 10744-10749, 2004.

[18] Y. Kitao, Y. Imai, K. Ozawa et al., "Pael receptor induces death of dopaminergic neurons in the substantia nigra via endoplasmic reticulum stress and dopamine toxicity, which is enhanced under condition of parkin inactivation," Human Molecular Genetics, vol. 16, no. 1, pp. 50-60, 2007.

[19] J. C. Greene, A. J. Whitworth, I. Kuo, L. A. Andrews, M. B. Feany, and L. J. Pallanck, "Mitochondrial pathology and apoptotic muscle degeneration in Drosophila parkin mutants," Proceedings of the National Academy of Sciences of the United States of America, vol. 100, no. 7, pp. 4078-4083, 2003.

[20] Y. Pesah, T. Pham, H. Burgess et al., "Drosophila parkin mutants have decreased mass and cell size and increased sensitivity to oxygen radical stress," Development, vol. 131, no. 9, pp. 2183-2194, 2004.

[21] Y. Yang, S. Gehrke, Y. Imai et al., "Mitochondrial pathology and muscle and dopaminergic neuron degeneration caused by inactivation of Drosophila Pink1 is rescued by Parkin," Proceedings of the National Academy of Sciences of the United States of America, vol. 103, no. 28, pp. 10793-10798, 2006.

[22] I. E. Clark, M. W. Dodson, C. Jiang et al., "Drosophila pink1 is required for mitochondrial function and interacts genetically with parkin," Nature, vol. 441, no. 7097, pp. 1162-1166, 2006.

[23] J. Park, S. B. Lee, S. Lee et al., "Mitochondrial dysfunction in Drosophila PINK1 mutants is complemented by parkin," Nature, vol. 441, no. 7097, pp. 1157-1161, 2006.

[24] A. C. Poole, R. E. Thomas, L. A. Andrews, H. M. McBride, A. J. Whitworth, and L. J. Pallanck, "The PINK1/Parkin pathway regulates mitochondrial morphology," Proceedings of the National Academy of Sciences of the United States of America, vol. 105, no. 5, pp. 1638-1643, 2008.

[25] Y. Yang, Y. Ouyang, L. Yang et al., "Pink1 regulates mitochondrial dynamics through interaction with the fission/fusion machinery," Proceedings of the National Academy of Sciences of the United States of America, vol. 105, no. 19, pp. 7070-7075, 2008.

[26] H. Deng, M. W. Dodson, H. Huang, and M. Guo, "The Parkinson's disease genes pink1 and parkin promote mitochondrial fission and/or inhibit fusion in Drosophila," Proceedings of the National Academy of Sciences of the United States of America, vol. 105, no. 38, pp. 14503-14508, 2008.

[27] Y. Imai, T. Kanao, T. Sawada et al., "The loss of PGAM5 suppresses the mitochondrial degeneration caused by inactivation of PINK1 in Drosophila," PLoS Genetics, vol. 6, no. 12, Article ID e1001229, 2010.

[28] W. Yu, Y. Sun, S. Guo, and B. Lu, "The PINK1/Parkin pathway regulates mitochondrial dynamics and function in mammalian hippocampal and dopaminergic neurons," Human Molecular Genetics, vol. 20, no. 16, pp. 3227-3240, 2011.

[29] D. Narendra, A. Tanaka, D. F. Suen, and R. J. Youle, "Parkin is recruited selectively to impaired mitochondria and promotes their autophagy," Journal of Cell Biology, vol. 183, no. 5, pp. 795-803, 2008.

[30] J. Y. Lee, Y. Nagano, J. P. Taylor, K. L. Lim, and T. P. Yao, "Disease-causing mutations in Parkin impair mitochondrial ubiquitination, aggregation, and HDAC6-dependent mitophagy," Journal of Cell Biology, vol. 189, no. 4, pp. 671$679,2010$.

[31] J. A. Olzmann, A. Li, M. V. Chudaev et al., "Parkin-mediated K63-linked polyubiquitination targets misfolded DJ-1 to aggresomes via binding to HDAC6," Journal of Cell Biology, vol. 178, no. 6, pp. 1025-1038, 2007.

[32] N. C. Chan, A. M. Salazar, A. H. Pham et al., "Broad activation of the ubiquitin-proteasome system by Parkin is critical for mitophagy," Human Molecular Genetics, vol. 20, no. 9, pp. 1726-1737, 2011.

[33] S. Geisler, K. M. Holmström, D. Skujat et al., "PINK1/Parkinmediated mitophagy is dependent on VDAC1 and p62/SQSTM1," Nature Cell Biology, vol. 12, no. 2, pp. 119-131, 2010. 
[34] K. Okatsu, K. Saisho, M. Shimanuki et al., "P62/SQSTM1 cooperates with Parkin for perinuclear clustering of depolarized mitochondria," Genes to Cells, vol. 15, no. 8, pp. 887-900, 2010.

[35] D. P. Narendra, L. A. Kane, D. N. Hauser, I. M. Fearnley, and R. J. Youle, "p62/SQSTM1 is required for Parkin-induced mitochondrial clustering but not mitophagy; VDAC1 is dispensable for both," Autophagy, vol. 6, no. 8, pp. 10901106, 2010.

[36] Y. Saeki, T. Kudo, T. Sone et al., "Lysine 63-linked polyubiquitin chain may serve as a targeting signal for the $26 \mathrm{~S}$ proteasome," EMBO Journal, vol. 28, no. 4, pp. 359-371, 2009.

[37] M. Komatsu, S. Waguri, M. Koike et al., "Homeostatic levels of p62 control cytoplasmic inclusion body formation in autophagy-deficient mice," Cell, vol. 131, no. 6, pp. 11491163, 2007.

[38] U. B. Pandey, Z. Nie, Y. Batlevi et al., "HDAC6 rescues neurodegeneration and provides an essential link between autophagy and the UPS," Nature, vol. 447, no. 7146, pp. 859-863, 2007.

[39] C. van Humbeeck, T. Cornelissen, H. Hofkens et al., "Parkin interacts with ambral to induce mitophagy," Journal of Neuroscience, vol. 31, no. 28, pp. 10249-10261, 2011.

[40] Y. Li, O. W. Wan, W. Xie, and K. K. Chung, "p32 regulates mitochondrial morphology and dynamics through parkin," Neuroscience, vol. 199, pp. 346-358, 2011.

[41] V. Fogal, A. D. Richardson, P. P. Karmali, I. E. Scheffler, J. W. Smith, and E. Ruoslahti, "Mitochondrial p32 protein is a critical regulator of tumor metabolism via maintenance of oxidative phosphorylation," Molecular and Cellular Biology, vol. 30, no. 6, pp. 1303-1318, 2010.

[42] V. A. Hristova, S. A. Beasley, R. J. Rylett, and G. S. Shaw, "Identification of a novel $\mathrm{Zn}^{2+}$-binding domain in the autosomal recessive juvenile Parkinson-related E3 ligase parkin," The Journal of Biological Chemistry, vol. 284, no. 22, pp. 14978-14986, 2009.

[43] C. Fernandes and Y. Rao, "Genome-wide screen for modifiers of Parkinson's disease genes in Drosophila," Molecular Brain, vol. 4, no. 1, article 17, 2011.

[44] T. Kanki, K. Wang, Y. Cao, M. Baba, and D. J. Klionsky, “Atg32 is a mitochondrial protein that confers selectivity during mitophagy," Developmental Cell, vol. 17, no. 1, pp. 98-109, 2009.

[45] K. Okamoto, N. Kondo-Okamoto, and Y. Ohsumi, "Mitochondria-anchored receptor Atg32 mediates degradation of mitochondria via selective autophagy," Developmental Cell, vol. 17, no. 1, pp. 87-97, 2009.

[46] G. Chinnadurai, S. Vijayalingam, and S. B. Gibson, "BNIP3 subfamily BH3-only proteins: mitochondrial stress sensors in normal and pathological functions," Oncogene, vol. 27, no. 1, pp. S114-S127, 2008.

[47] A. Diwan, S. J. Matkovich, Q. Yuan et al., "Endoplasmic reticulum-mitochondria crosstalk in NIX-mediated murine cell death," Journal of Clinical Investigation, vol. 119, no. 1, pp. 203-212, 2009.

[48] K. Fujimoto, E. L. Ford, H. Tran et al., "Loss of Nix in Pdx1deficient mice prevents apoptotic and necrotic $\beta$ cell death and diabetes," Journal of Clinical Investigation, vol. 120, no. 11, pp. 4031-4039, 2010.

[49] H. Sandoval, P. Thiagarajan, S. K. Dasgupta et al., "Essential role for Nix in autophagic maturation of erythroid cells," Nature, vol. 454, no. 7201, pp. 232-235, 2008.
[50] I. Novak, V. Kirkin, D. G. McEwan et al., "Nix is a selective autophagy receptor for mitochondrial clearance," EMBO Reports, vol. 11, no. 1, pp. 45-51, 2010.

[51] W. X. Ding, H. M. Ni, M. Li et al., "Nix is critical to two distinct phases of mitophagy, reactive oxygen species-mediated autophagy induction and Parkin-ubiquitin-p62-mediated mitochondrial priming," The Journal of Biological Chemistry, vol. 285, no. 36, pp. 27879-27890, 2010.

[52] R. L. Schweers, J. Zhang, M. S. Randall et al., "NIX is required for programmed mitochondrial clearance during reticulocyte maturation," Proceedings of the National Academy of Sciences of the United States of America, vol. 104, no. 49, pp. 19500-19505, 2007.

[53] N. Kitamura, Y. Nakamura, Y. Miyamoto et al., "Mieap, a p53-inducible protein, controls mitochondrial quality by repairing or eliminating unhealthy mitochondria," PLoS ONE, vol. 6, no. 1, Article ID e16060, 2011.

[54] Y. Miyamoto, N. Kitamura, Y. Nakamura et al., "Possible existence of lysosome-like organella within mitochondria and its role in mitochondrial quality control," PLoS ONE, vol. 6, no. 1, Article ID e16054, 2011.

[55] C. Vives-Bauza, C. Zhou, Y. Huang et al., "PINK1-dependent recruitment of Parkin to mitochondria in mitophagy," Proceedings of the National Academy of Sciences of the United States of America, vol. 107, no. 1, pp. 378-383, 2010.

[56] S. Kawajiri, S. Saiki, S. Sato et al., "PINK1 is recruited to mitochondria with parkin and associates with LC3 in mitophagy," FEBS Letters, vol. 584, no. 6, pp. 1073-1079, 2010.

[57] N. Matsuda, S. Sato, K. Shiba et al., "PINK1 stabilized by mitochondrial depolarization recruits Parkin to damaged mitochondria and activates latent Parkin for mitophagy," Journal of Cell Biology, vol. 189, no. 2, pp. 211-221, 2010.

[58] D. P. Narendra, S. M. Jin, A. Tanaka et al., "PINK1 is selectively stabilized on impaired mitochondria to activate Parkin," PLoS Biology, vol. 8, no. 1, Article ID e1000298, 2010.

[59] E. Ziviani, R. N. Tao, and A. J. Whitworth, "Drosophila Parkin requires PINK1 for mitochondrial translocation and ubiquitinates Mitofusin," Proceedings of the National Academy of Sciences of the United States of America, vol. 107, no. 11, pp. 5018-5023, 2010.

[60] P. Seibler, J. Graziotto, H. Jeong, F. Simunovic, C. Klein, and D. Krainc, "Mitochondrial parkin recruitment is impaired in neurons derived from mutant PINK1 induced pluripotent stem cells," Journal of Neuroscience, vol. 31, no. 16, pp. 59705976, 2011.

[61] A. Tanaka, M. M. Cleland, S. Xu et al., "Proteasome and p97 mediate mitophagy and degradation of mitofusins induced by Parkin," Journal of Cell Biology, vol. 191, no. 7, pp. 13671380, 2010.

[62] M. E. Gegg, J. M. Cooper, K. Y. Chau, M. Rojo, A. H. V. Schapira, and J. W. Taanman, "Mitofusin 1 and mitofusin 2 are ubiquitinated in a PINK1/parkin-dependent manner upon induction of mitophagy," Human Molecular Genetics, vol. 19, no. 24, pp. 4861-4870, 2010.

[63] H. Wang, P. Song, L. Du et al., "Parkin ubiquitinates Drp1 for proteasome-dependent degradation: implication of dysregulated mitochondrial dynamics in Parkinson disease," The Journal of Biological Chemistry, vol. 286, no. 13, pp. 1164911658, 2011.

[64] D. Chen, F. Gao, B. Li et al., "Parkin mono-ubiquitinates Bcl2 and regulates autophagy," The Journal of Biological Chemistry, vol. 285, no. 49, pp. 38214-38223, 2010. 
[65] A. C. Poole, R. E. Thomas, S. Yu, E. S. Vincow, and L. Pallanck, "The mitochondrial fusion-promoting factor mitofusin is a substrate of the PINK1/parkin pathway," PLoS ONE, vol. 5, no. 4, Article ID e10054, 2010.

[66] M. Unoki and Y. Nakamura, "Growth-suppressive effects of $\mathrm{BPOZ}$ and EGR2, two genes involved in the PTEN signaling pathway," Oncogene, vol. 20, no. 33, pp. 4457-4465, 2001.

[67] Y. Mei, Y. Zhang, K. Yamamoto, W. Xie, T. W. Mak, and H. You, "FOXO3a-dependent regulation of Pink1 (Park6) mediates survival signaling in response to cytokine deprivation," Proceedings of the National Academy of Sciences of the United States of America, vol. 106, no. 13, pp. 5153-5158, 2009.

[68] A. J. Whitworth, J. R. Lee, V. M. W. Ho, R. Flick, R. Chowdhury, and G. A. McQuibban, "Rhomboid-7 and HtrA2/Omi act in a common pathway with the Parkinson's disease factors Pink1 and Parkin," DMM Disease Models and Mechanisms, vol. 1, no. 2-3, pp. 168-174, 2008.

[69] E. Deas, H. Plun-Favreau, S. Gandhi et al., "PINK1 cleavage at position $\mathrm{A} 103$ by the mitochondrial protease PARL," Human Molecular Genetics, vol. 20, no. 5, pp. 867-879, 2011.

[70] S. M. Jin, M. Lazarou, C. Wang, L. A. Kane, D. P. Narendra, and R. J. Youle, "Mitochondrial membrane potential regulates PINK1 import and proteolytic destabilization by PARL," Journal of Cell Biology, vol. 191, no. 5, pp. 933-942, 2010.

[71] C. Meissner, H. Lorenz, A. Weihofen, D. J. Selkoe, and M. K. Lemberg, "The mitochondrial intramembrane protease PARL cleaves human Pink1 to regulate Pink1 trafficking," Journal of Neurochemistry, vol. 117, no. 5, pp. 856-867, 2011.

[72] G. Shi, J. R. Lee, D. A. Grimes et al., "Functional alteration of PARL contributes to mitochondrial dysregulation in Parkinson's disease," Human Molecular Genetics, vol. 20, no. 10, Article ID ddr077, pp. 1966-1974, 2011.

[73] M. Lazarou, S. M. Jin, L. A. Kane, and R. J. Youle, "Role of PINK1 binding to the TOM complex and alternate intracellular membranes in recruitment and activation of the E3 ligase Parkin," Developmental Cell, vol. 22, no. 2, pp. 320-333, 2012.

[74] C. Zhou, Y. Huang, Y. Shao et al., "The kinase domain of mitochondrial PINK1 faces the cytoplasm," Proceedings of the National Academy of Sciences of the United States of America, vol. 105, no. 33, pp. 12022-12027, 2008.

[75] K. Okatsu, T. Oka, M. Iguchi et al., "PINK1 autophosphorylation upon membrane potential dissipation is essential for Parkin recruitment to damaged mitochondria," Nature Communications, vol. 3, Article ID 1016, 2012.

[76] K. Shiba-Fukushima, Y. Imai, S. Yoshida et al., "PINK1mediated phosphorylation of the Parkin ubiquitin-like domain primes mitochondrial translocation of Parkin: an initial step of mitophagy," in Proceedings of the Meeting of Brain Environment supported by Grant-in-Aid for Scientific Research on Innovative Areas, Sendai, Japan, July 2012.

[77] C. Kondapalli, A. Kazlauskaite, N. Zhang et al., "PINK1 is activated by mitochondrial membrane potential depolarization and stimulates Parkin E3 ligase activity by phosphorylating Serine 65," Open Biology, vol. 2, no. 5, Article ID 120080, 2012.

[78] H. Q. Wang, Y. Imai, A. Kataoka, and R. Takahashi, "Cell type-specific upregulation of parkin in response to ER stress," Antioxidants and Redox Signaling, vol. 9, no. 5, pp. 533-542, 2007.

[79] Y. Imai, M. Soda, S. Hatakeyama et al., "CHIP is associated with Parkin, a gene responsible for familial Parkinson's Disease, and enhances its ubiquitin ligase activity," Molecular Cell, vol. 10, no. 1, pp. 55-67, 2002.
[80] Y. Imai, M. Soda, H. Inoue, N. Hattori, Y. Mizuno, and R. Takahashi, "An unfolded putative transmembrane polypeptide, which can lead to endoplasmic reticulum stress, is a substrate of Parkin," Cell, vol. 105, no. 7, pp. 891-902, 2001.

[81] L. Bouman, A. Schlierf, A. K. Lutz et al., "Parkin is transcriptionally regulated by ATF4: evidence for an interconnection between mitochondrial stress and ER stress," Cell Death and Differentiation, vol. 18, no. 5, pp. 769-782, 2011.

[82] Y. Higashi, M. Asanuma, I. Miyazaki, N. Hattori, Y. Mizuno, and N. Ogawa, "Parkin attenuates manganese-induced dopaminergic cell death," Journal of Neurochemistry, vol. 89, no. 6, pp. 1490-1497, 2004.

[83] Y. X. Yang, M. M. K. Muqit, and D. S. Latchman, "Induction of parkin expression in the presence of oxidative stress," European Journal of Neuroscience, vol. 24, no. 5, pp. 13661372, 2006.

[84] C. Zhang, M. Lin, R. Wu et al., "Parkin, a p53 target gene, mediates the role of p53 in glucose metabolism and the Warburg effect," Proceedings of the National Academy of Sciences United States of America, vol. 108, no. 39, pp. 16259-16264, 2011.

[85] C. A. da Costa, C. Sunyach, E. Giaime et al., "Transcriptional repression of $\mathrm{p} 53$ by parkin and impairment by mutations associated with autosomal recessive juvenile Parkinson's disease," Nature Cell Biology, vol. 11, no. 11, pp. 1370-1375, 2009.

[86] S. R. Denison, G. Callahan, N. A. Becker, L. A. Phillips, and D. I. Smith, "Characterization of FRA6E and its potential role in autosomal recessive juvenile parkinsonism and ovarian cancer," Genes Chromosomes and Cancer, vol. 38, no. 1, pp. 40-52, 2003.

[87] S. R. Denison, F. Wang, N. A. Becker et al., "Alterations in the common fragile site gene Parkin in ovarian and other cancers," Oncogene, vol. 22, no. 51, pp. 8370-8378, 2003.

[88] S. J. Mehdi, A. Ali, and M. M. A. Rizvi, "Parkin gene alterations in ovarian carcinoma from northern indian population," Pathology and Oncology Research, vol. 17, no. 3, pp. 579-586, 2011.

[89] A. Letessier, S. Garrido-Urbani, C. Ginestier et al., "Correlated break at PARK2/FRA6E and loss of AF-6/Afadin protein expression are associated with poor outcome in breast cancer," Oncogene, vol. 26, no. 2, pp. 298-307, 2007.

[90] X. Agirre, J. Román-Gómez, I. Vázquez et al., "Abnormal methylation of the common PARK2 and PACRG promoter is associated with downregulation of gene expression in acute lymphoblastic leukemia and chronic myeloid leukemia," International Journal of Cancer, vol. 118, no. 8, pp. 19451953, 2006.

[91] K. Ikeuchi, H. Marusawa, M. Fujiwara et al., "Attenuation of proteolysis-mediated cyclin E regulation by alternatively spliced Parkin in human colorectal cancers," International Journal of Cancer, vol. 125, no. 9, pp. 2029-2035, 2009.

[92] M. C. Picchio, E. S. Martin, R. Cesari et al., "Alterations of the tumor suppressor gene parkin in non-small cell lung cancer," Clinical Cancer Research, vol. 10, no. 8, pp. 2720-2724, 2004.

[93] S. Veeriah, B. S. Taylor, S. Meng et al., "Somatic mutations of the Parkinson's disease-associated gene PARK2 in glioblastoma and other human malignancies," Nature Genetics, vol. 42, no. 1, pp. 77-82, 2010.

[94] R. Cesari, E. S. Martin, G. A. Calin et al., "Parkin, a gene implicated in autosomal recessive juvenile parkinsonism, is a candidate tumor suppressor gene on chromosome 6q25q27," Proceedings of the National Academy of Sciences of the 
United States of America, vol. 100, no. 10, pp. 5956-5961, 2003.

[95] M. Fujiwara, H. Marusawa, H. Q. Wang et al., "Parkin as a tumor suppressor gene for hepatocellular carcinoma," Oncogene, vol. 27, no. 46, pp. 6002-6011, 2008.

[96] F. Wang, S. Denison, J. P. Lai et al., "Parkin gene alterations in hepatocellular carcinoma," Genes Chromosomes and Cancer, vol. 40, no. 2, pp. 85-96, 2004.

[97] A. Fransson, A. Ruusala, and P. Aspenström, "Atypical Rho GTPases have roles in mitochondrial homeostasis and apoptosis," The Journal of Biological Chemistry, vol. 278, no. 8, pp. 6495-6502, 2003.

[98] L. Giot, J. S. Bader, C. Brouwer et al., "A protein interaction map of Drosophila melanogaster," Science, vol. 302, no. 5651, pp. 1727-1736, 2003.

[99] X. Guo, G. T. Macleod, A. Wellington et al., "The GTPase dMiro is required for axonal transport of mitochondria to Drosophila synapses," Neuron, vol. 47, no. 3, pp. 379-393, 2005.

[100] E. E. Glater, L. J. Megeath, R. S. Stowers, and T. L. Schwarz, "Axonal transport of mitochondria requires milton to recruit kinesin heavy chain and is light chain independent," Journal of Cell Biology, vol. 173, no. 4, pp. 545-557, 2006.

[101] A. Weihofen, K. J. Thomas, B. L. Ostaszewski, M. R. Cookson, and D. J. Selkoe, "Pink1 forms a multiprotein complex with miro and milton, linking Pink1 function to mitochondrial trafficking," Biochemistry, vol. 48, no. 9, pp. 2045-2052, 2009.

[102] S. Liu, T. Sawada, S. Lee et al., "Parkinson's disease-associated kinase PINK1 regulates Miro protein level and axonal transport of mitochondria," PLoS Genet, vol. 8, no. 3, Article ID e1002537, 2012.

[103] X. Wang, D. Winter, G. Ashrafi et al., "PINK1 and parkin target miro for phosphorylation and degradation to arrest mitochondrial motility," Cell, vol. 147, no. 4, pp. 893-906, 2011.

[104] V. Bonifati, P. Rizzu, M. J. Van Baren et al., "Mutations in the DJ-1 gene associated with autosomal recessive early-onset parkinsonism," Science, vol. 299, no. 5604, pp. 256-259, 2003.

[105] T. Taira, Y. Saito, T. Niki, S. M. M. Iguchi-Ariga, K. Takahashi, and $\mathrm{H}$. Ariga, "DJ-1 has a role in antioxidative stress to prevent cell death," EMBO Reports, vol. 5, no. 2, pp. 213-218, 2004.

[106] F. M. Menzies, S. C. Yenisetti, and K. T. Min, "Roles of Drosophila DJ-1 in survival of dopaminergic neurons and oxidative stress," Current Biology, vol. 15, no. 17, pp. 1578-1582, 2005.

[107] M. Meulener, A. J. Whitworth, C. E. Armstrong-Gold et al., "Drosophila DJ-1 mutants are selectively sensitive to environmental toxins associated with Parkinson's disease," Current Biology, vol. 15, no. 17, pp. 1572-1577, 2005.

[108] Y. Yang, S. Gehrke, M. E. Haque et al., "Inactivation of Drosophila DJ-1 leads to impairments of oxidative stress response and phosphatidylinositol 3-kinase/Akt signaling," Proceedings of the National Academy of Sciences of the United States of America, vol. 102, no. 38, pp. 13670-13675, 2005.

[109] C. Martinat, S. Shendelman, A. Jonason et al., "Sensitivity to oxidative stress in DJ-1-deficient dopamine neurons: an ESderived cell model of primary Parkinsonism," PLoS Biology, vol. 2, no. 11, 2004.

[110] I. Irrcher, H. Aleyasin, E. L. Seifert et al., "Loss of the Parkinson's disease-linked gene DJ-1 perturbs mitochondrial dynamics," Human Molecular Genetics, vol. 19, no. 19, pp. 3734-3746, 2010.
[111] K. J. Thomas, M. K. McCoy, J. Blackinton et al., "DJ-1 acts in parallel to the PINK1/parkin pathway to control mitochondrial function and autophagy," Human Molecular Genetics, vol. 20, no. 1, pp. 40-50, 2011.

[112] L. Y. Hao, B. I. Giasson, and N. M. Bonini, "DJ-1 is critical for mitochondrial function and rescues PINK1 loss of function," Proceedings of the National Academy of Sciences of the United States of America, vol. 107, no. 21, pp. 9747-9752, 2010.

[113] T. Kitada, Y. Tong, C. A. Gautier, and J. Shen, "Absence of nigral degeneration in aged parkin/DJ-1/PINK1 triple knockout mice," Journal of Neurochemistry, vol. 111, no. 3, pp. 696-702, 2009.

[114] A. P. Joselin, S. J. Hewitt, S. M. Callaghan et al., "ROS dependent regulation of Parkin and DJ-1 localization during oxidative stress in neurons," Human Molecular Genetics, vol. 21, no. 22, pp. 4888-4903, 2012.

[115] A. J. Whitworth, D. A. Theodore, J. C. Greene, H. Beneš, P. D. Wes, and L. J. Pallanck, "Increased glutathione S-transferase activity rescues dopaminergic neuron loss in a Drosophila model of Parkinson's disease," Proceedings of the National Academy of Sciences of the United States of America, vol. 102, no. 22, pp. 8024-8029, 2005.

[116] Y. Suzuki, Y. Imai, H. Nakayama, K. Takahashi, K. Takio, and R. Takahashi, "A serine protease, HtrA2, is released from the mitochondria and interacts with XIAP, inducing cell death," Molecular Cell, vol. 8, no. 3, pp. 613-621, 2001.

[117] K. Sekine, Y. Hao, Y. Suzuki, R. Takahashi, T. Tsuruo, and M. Naito, "HtrA2 cleaves Apollon and induces cell death by IAP-binding motif in Apollon-deficient cells," Biochemical and Biophysical Research Communications, vol. 330, no. 1, pp. 279-285, 2005.

[118] L. M. Martins, I. Iaccarino, T. Tenev et al., "The serine protease Omi/HtrA2 regulates apoptosis by binding XIAP through a Reaper-like motif," The Journal of Biological Chemistry, vol. 277, no. 1, pp. 439-444, 2002.

[119] G. van Loo, M. van Gurp, B. Depuydt et al., "The serine protease Omi/HtrA2 is released from mitochondria during apoptosis. Omi interacts with caspase-inhibitor XIAP and induces enhanced caspase activity," Cell Death and Differentiation, vol. 9, no. 1, pp. 20-26, 2002.

[120] K. M. Strauss, L. M. Martins, H. Plun-Favreau et al., "Loss of function mutations in the gene encoding Omi/HtrA2 in Parkinson's disease," Human Molecular Genetics, vol. 14, no. 15, pp. 2099-2111, 2005.

[121] V. Bogaerts, K. Nuytemans, J. Reumers et al., "Genetic variability in the mitochondrial serine protease HTRA2 contributes to risk for Parkinson disease," Human Mutation, vol. 29, no. 6, pp. 832-840, 2008.

[122] J. Simón-Sánchez and A. B. Singleton, "Sequencing analysis of OMI/HTRA2 shows previously reported pathogenic mutations in neurologically normal controls," Human Molecular Genetics, vol. 17, no. 13, pp. 1988-1993, 2008.

[123] J. M. Jones, P. Datta, S. M. Srinivasula et al., "Loss of Omi mitochondrial protease activity causes the neuromuscular disorder of mnd2 mutant mice," Nature, vol. 425, no. 6959, pp. 721-727, 2003.

[124] H. Plun-Favreau, K. Klupsch, N. Moisoi et al., "The mitochondrial protease HtrA2 is regulated by Parkinson's diseaseassociated kinase PINK1," Nature Cell Biology, vol. 9, no. 11, pp. 1243-1252, 2007.

[125] H. M. Park, G. Y. Kim, M. K. Nam et al., "The serine protease HtrA2/Omi cleaves Parkin and irreversibly inactivates its E3 ubiquitin ligase activity," Biochemical and Biophysical Research Communications, vol. 387, no. 3, pp. 537-542, 2009. 
[126] J. Yun, J. H. Cao, M. W. Dodson et al., "Loss-of-function analysis suggests that Omi/HtrA2 is not an essential component of the pink1/parkin pathway in vivo," Journal of Neuroscience, vol. 28, no. 53, pp. 14500-14510, 2008.

[127] T. Yoshida, T. Mizuta, and S. Shimizu, "Neurodegeneration in mnd2 mutant mice is not prevented by parkin transgene," Biochemical and Biophysical Research Communications, vol. 402, no. 4, pp. 676-679, 2010.

[128] D. F. Suen, D. P. Narendra, A. Tanaka, G. Manfredi, and R. J. Youle, "Parkin overexpression selects against a deleterious mtDNA mutation in heteroplasmic cybrid cells," Proceedings of the National Academy of Sciences of the United States of America, vol. 107, no. 26, pp. 11835-11840, 2010.

[129] A. Rakovic, A. Grünewald, J. Kottwitz et al., "Mutations in PINK1 and Parkin impair ubiquitination of Mitofusins in human fibroblasts," PLOS ONE, vol. 6, no. 3, Article ID e16746, 2011.

[130] S. Gispert, F. Ricciardi, A. Kurz et al., "Parkinson phenotype in aged PINK1-deficient mice is accompanied by progressive mitochondrial dysfunction in absence of neurodegeneration," PLoS ONE, vol. 4, no. 6, Article ID e5777, 2009.

[131] C. A. Gautier, T. Kitada, and J. Shen, "Loss of PINK1 causes mitochondrial functional defects and increased sensitivity to oxidative stress," Proceedings of the National Academy of Sciences of the United States of America, vol. 105, no. 32, pp. 11364-11369, 2008.

[132] S. Schmidt, B. Linnartz, S. Mendritzki et al., "Genetic mouse models for Parkinson's disease display severe pathology in glial cell mitochondria," Human Molecular Genetics, vol. 20, no. 6, pp. 1197-1211, 2011.

[133] F. Billia, L. Hauck, F. Konecny, V. Rao, J. Shen, and T. W. Mak, "PTEN-inducible kinase 1 (PINK1)/Park6 is indispensable for normal heart function," Proceedings of the National Academy of Sciences of the United States of America, vol. 108, no. 23, pp. 9572-9577, 2011.

[134] C. Huang, A. M. Andres, E. P. Ratliff, G. Hernandez, P. Lee, and R. A. Gottlieb, "Preconditioning involves selective mitophagy mediated by parkin and p62/SQSTM1," PLoS ONE, vol. 6, no. 6, Article ID e20975, 2011.

[135] T. A. Zesiewicz, J. A. Strom, A. R. Borenstein et al., "Heart failure in Parkinson's disease: analysis of the United States medicare current beneficiary survey," Parkinsonism and Related Disorders, vol. 10, no. 7, pp. 417-420, 2004.

[136] A. Anvret, C. Ran, M. Westerlund et al., "Genetic screening of the mitochondrial Rho gtpases MIRO1 and MIRO2 in Parkinson's disease," Open Neurology Journal, vol. 6, pp. 15, 2012.

[137] A. H. Pham, S. Meng, Q. N. Chu, and D. C. Chan, "Loss of Mfn2 results in progressive, retrograde degeneration of dopaminergic neurons in the nigrostriatal circuit," Human Molecular Genetics, vol. 21, no. 22, pp. 4827-4835, 2012.

[138] S. Lee, F. H. Sterky, A. Mourier et al., "Mitofusin 2 is necessary for striatal axonal projections of midbrain dopamine neurons," Human Molecular Genetics, vol. 21, no. 22, pp. 4827-4835, 2012.

[139] K. Verhoeven, K. G. Claeys, S. Züchner et al., "MFN2 mutation distribution and genotype/phenotype correlation in Charcot-Marie-Tooth type 2," Brain, vol. 129, no. 8, pp. 2093-2102, 2006.

[140] V. H. Lawson, B. V. Graham, and K. M. Flanigan, "Clinical and electrophysiologic features of CMT2A with mutations in the mitofusin 2 gene," Neurology, vol. 65, no. 2, pp. 197-204, 2005.
[141] S. Züchner, I. V. Mersiyanova, M. Muglia et al., "Mutations in the mitochondrial GTPase mitofusin 2 cause Charcot-MarieTooth neuropathy type 2A," Nature Genetics, vol. 36, no. 5, pp. 449-451, 2004.

[142] A. Misko, S. Jiang, I. Wegorzewska, J. Milbrandt, and R. $\mathrm{H}$. Baloh, "Mitofusin 2 is necessary for transport of axonal mitochondria and interacts with the Miro/Milton complex," Journal of Neuroscience, vol. 30, no. 12, pp. 4232-4240, 2010.

[143] J. J. Palacino, D. Sagi, M. S. Goldberg et al., "Mitochondrial dysfunction and oxidative damage in parkin-deficient mice," The Journal of Biological Chemistry, vol. 279, no. 18, pp. 18614-18622, 2004.

[144] V. A. Morais, P. Verstreken, A. Roethig et al., "Parkinson's disease mutations in PINK1 result in decreased complex I activity and deficient synaptic function," EMBO Molecular Medicine, vol. 1, no. 2, pp. 99-111, 2009.

[145] L. Flinn, H. Mortiboys, K. Volkmann, R. W. Kster, P. W. Ingham, and O. Bandmann, "Complex I deficiency and dopaminergic neuronal cell loss in parkin-deficient zebrafish (Danio rerio)," Brain, vol. 132, no. 6, pp. 1613-1623, 2009.

[146] H. Mortiboys, K. J. Thomas, W. J. H. Koopman et al., "Mitochondrial function and morphology are impaired in parkinmutant fibroblasts," Annals of Neurology, vol. 64, no. 5, pp. 555-565, 2008.

[147] T. Amo, S. Sato, S. Saiki et al., "Mitochondrial membrane potential decrease caused by loss of PINK1 is not due to proton leak, but to respiratory chain defects," Neurobiology of Disease, vol. 41, no. 1, pp. 111-118, 2011.

[148] S. Gandhi, A. Wood-Kaczmar, Z. Yao et al., "PINK1-Associated Parkinson's disease is caused by neuronal vulnerability to calcium-induced cell death," Molecular Cell, vol. 33, no. 5, pp. 627-638, 2009.

[149] S. Vilain, G. Esposito, D. Haddad et al., "The yeast complex I equivalent NADH dehydrogenase rescues pink1 mutants," PLoS Genet, vol. 8, no. 1, Article ID e1002456, 2012.

[150] M. Vos, G. Esposito, J. N. Edirisinghe et al., "Vitamin K2 is a mitochondrial electron carrier that rescues pink1 deficiency," Science, vol. 336, no. 6086, pp. 1306-1310, 2012.

[151] B. M. Zid, A. N. Rogers, S. D. Katewa et al., "4E-BP extends lifespan upon dietary restriction by enhancing mitochondrial activity in Drosophila," Cell, vol. 139, no. 1, pp. 149-160, 2009.

[152] S. Liu and B. Lu, "Reduction of protein translation and activation of autophagy protect against PINK1 pathogenesis in Drosophila melanogaster," PLoS Genetics, vol. 6, no. 12, p. e1001237, 2010.

[153] L. S. Tain, H. Mortiboys, R. N. Tao, E. Ziviani, O. Bandmann, and A. J. Whitworth, "Rapamycin activation of 4E-BP prevents parkinsonian dopaminergic neuron loss," Nature Neuroscience, vol. 12, no. 9, pp. 1129-1135, 2009.

[154] J. H. Shin, H. S. Ko, H. Kang et al., "PARIS (ZNF746) repression of PGC- $1 \alpha$ contributes to neurodegeneration in parkinson's disease," Cell, vol. 144, no. 5, pp. 689-702, 2011.

[155] C. Pacelli, D. De Rasmo, A. Signorile et al., "Mitochondrial defect and PGC- $1 \alpha$ dysfunction in parkin-associated familial Parkinson's disease," Biochimica et Biophysica Acta, vol. 1812, no. 8, pp. 1041-1053, 2011.

[156] A. W. Greene, K. Grenier, M. A. Aguileta et al., "Mitochondrial processing peptidase regulates PINK1 processing, import and Parkin recruitment," EMBO Reports, vol. 13, no. 4, pp. 378-385, 2012. 
[157] J. M. Bravo-San Pedro, R. Gomez-Sanchez, M. Niso-Santano et al., "The MAPK1/3 pathway is essential for the deregulation of autophagy observed in G2019S LRRK2 mutant fibroblasts," Autophagy, vol. 8, no. 10, pp. 1537-1539, 2012.

[158] J. M. Bravo-San Pedro, M. Niso-Santano, R. Gomez-Sanchez et al., "The LRRK2 G2019S mutant exacerbates basal autophagy through activation of the MEK/ERK pathway," Cellular and Molecular Life Sciences https://secure.juntendo .ac.jp/. In press.

[159] L. G. Friedman, M. L. Lachenmayer, J. Wang et al., "Disrupted autophagy leads to dopaminergic axon and dendrite degeneration and promotes presynaptic accumulation of alpha-synuclein and LRRK2 in the brain," Journal of Neuroscience, vol. 32, no. 22, pp. 7585-7593, 2012.

[160] P. Gomez-Suaga and S. Hilfiker, "LRRK2 as a modulator of lysosomal calcium homeostasis with downstream effects on autophagy," Autophagy, vol. 8, no. 4, pp. 692-693, 2012.

[161] E. D. Plowey, S. J. Cherra, Y. J. Liu, and C. T. Chu, "Role of autophagy in G2019S-LRRK2-associated neurite shortening in differentiated SH-SY5Y cells," Journal of Neurochemistry, vol. 105, no. 3, pp. 1048-1056, 2008.

[162] J. Alegre-Abarrategui, H. Christian, M. M. P. Lufino et al., "LRRK2 regulates autophagic activity and localizes to specific membrane microdomains in a novel human genomic reporter cellular model," Human Molecular Genetics, vol. 18, no. 21, pp. 4022-4034, 2009.

[163] J. P. Covy, E. A. Waxman, and B. I. Giasson, "Characterization of cellular protective effects of ATP13A2/PARK9 expression and alterations resulting from pathogenic mutants," Journal of Neuroscience Research, vol. 90, no. 12, pp. 2306-2316, 2012.

[164] A. Ramirez, A. Heimbach, J. Gründemann et al., "Hereditary parkinsonism with dementia is caused by mutations in ATP13A2, encoding a lysosomal type 5 P-type ATPase," Nature Genetics, vol. 38, no. 10, pp. 1184-1191, 2006.

[165] M. Usenovic, E. Tresse, J. R. Mazzulli, J. P. Taylor, and D. Krainc, "Deficiency of ATP13A2 leads to lysosomal dysfunction, alpha-synuclein accumulation, and neurotoxicity," Journal of Neuroscience, vol. 32, no. 12, pp. 4240-4246, 2012.

[166] B. Dehay, A. Ramirez, M. Martinez-Vicente et al., "Loss of P-type ATPase ATP13A2/PARK9 function induces general lysosomal deficiency and leads to Parkinson disease neurodegeneration," Proceedings of the National Academy of Sciences United States of America, vol. 109, no. 24, pp. 9611-9616, 2012.

[167] N. Moisoi, K. Klupsch, V. Fedele et al., "Mitochondrial dysfunction triggered by loss of HtrA2 results in the activation of a brain-specific transcriptional stress response," Cell Death and Differentiation, vol. 16, no. 3, pp. 449-464, 2009.

[168] B. Li, Q. Hu, H. Wang et al., "Omi/HtrA2 is a positive regulator of autophagy that facilitates the degradation of mutant proteins involved in neurodegenerative diseases," Cell Death and Differentiation, vol. 17, no. 11, pp. 1773-1784, 2010.

[169] A. D. Fonzo, M. C. J. Dekker, P. Montagna et al., "FBXO7 mutations cause autosomal recessive, early-onset parkinsonian- pyramidal syndrome," Neurology, vol. 72, no. 3, pp. 240 245, 2009.

[170] T. Zhao, E. de Graaff, G. J. Breedveld et al., "Loss of nuclear activity of the $\mathrm{FBXO} 7$ protein in patients with parkinsonianpyramidal syndrome (PARK15)," PLoS ONE, vol. 6, no. 2, Article ID e16983, 2011.

[171] R. Krüger, W. Kuhn, T. Müller et al., "Ala30Pro mutation in the gene encoding $\alpha$-synuclein in Parkinson's disease," Nature Genetics, vol. 18, no. 2, pp. 106-108, 1998.
[172] M. G. Spillantini, M. L. Schmidt, V. M. Y. Lee, J. Q. Trojanowski, R. Jakes, and M. Goedert, " $\alpha$-synuclein in Lewy bodies," Nature, vol. 388, no. 6645, pp. 839-840, 1997.

[173] M. H. Polymeropoulos, C. Lavedan, E. Leroy et al., "Mutation in the $\alpha$-synuclein gene identified in families with Parkinson's disease," Science, vol. 276, no. 5321, pp. 2045-2047, 1997.

[174] K. Arima, S. Hirai, N. Sunohara et al., "Cellular colocalization of phosphorylated tau- and NACP/ $\alpha$-synucleinepitopes in Lewy bodies in sporadic Parkinson's disease and in dementia with Lewy bodies," Brain Research, vol. 843, no. 1-2, pp. 53-61, 1999.

[175] P. T. Kotzbauer, B. I. Giasson, A. V. Kravitz et al., "Fibrillization of $\alpha$-synuclein and tau in familial Parkinson's disease caused by the A53T $\alpha$-synuclein mutation," Experimental Neurology, vol. 187, no. 2, pp. 279-288, 2004.

[176] T. Duka, V. Duka, J. N. Joyce, and A. Sidhu, " $\alpha$-Synuclein contributes to GSK-3 $\beta$-catalyzed Tau phosphorylation in Parkinson's disease models," FASEB Journal, vol. 23, no. 9, pp. 2820-2830, 2009.

[177] M. Hong, M. Hong, V. Zhukareva et al., "Mutation-specific functional impairments in distinct tau isoforms of hereditary FTDP-17,” Science, vol. 282, no. 5395, pp. 1914-1917, 1998.

[178] M. Hutton, C. L. Lendon, P. Rizzu et al., "Association of missense and 5'-splice-site mutations in tau with the inherited dementia FTDP-17," Nature, vol. 393, no. 6686, pp. 702-705, 1998.

[179] W. Satake, Y. Nakabayashi, I. Mizuta et al., "Genome-wide association study identifies common variants at four loci as genetic risk factors for Parkinson's disease," Nature Genetics, vol. 41, no. 12, pp. 1303-1307, 2009.

[180] J. Simon-Sanchez, C. Schulte, J. M. Bras et al., "Genome-wide association study reveals genetic risk underlying Parkinson's disease," Nature Genetics, vol. 41, no. 12, pp. 1308-1312, 2009.

[181] K. Takeda, T. Yoshida, S. Kikuchi et al., "Synergistic roles of the proteasome and autophagy for mitochondrial maintenance and chronological lifespan in fission yeast," Proceedings of the National Academy of Sciences of the United States of America, vol. 107, no. 8, pp. 3540-3545, 2010.

[182] I. Pimenta de Castro, A. C. Costa, D. Lam et al., "Genetic analysis of mitochondrial protein misfolding in Drosophila melanogaster," Cell Death \& Differentiation, vol. 19, no. 8, pp. 1308-1316, 2012.

[183] H. Meyer, M. Bug, and S. Bremer, "Emerging functions of the VCP/p97 AAA-ATPase in the ubiquitin system," Nature Cell Biology, vol. 14, no. 2, pp. 117-123, 2012.

[184] S. R. Yoshii, C. Kishi, N. Ishihara, and N. Mizushima, "Parkin mediates proteasome-dependent protein degradation and rupture of the outer mitochondrial membrane," The Journal of Biological Chemistry, vol. 286, no. 22, pp. 19630-19640, 2011. 

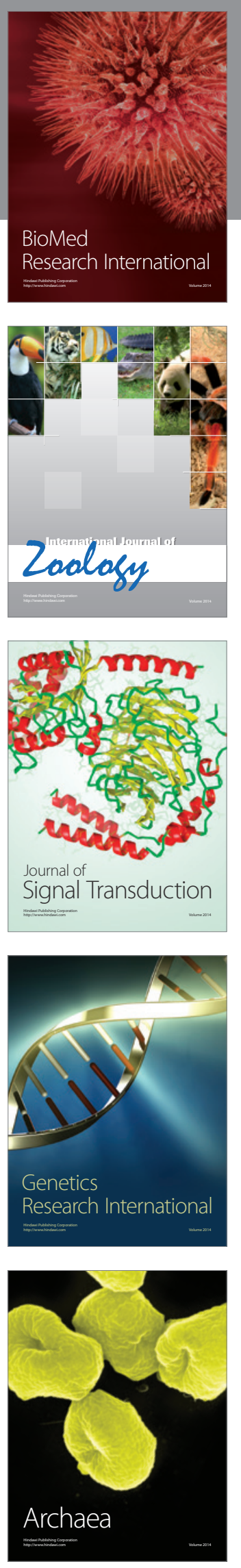
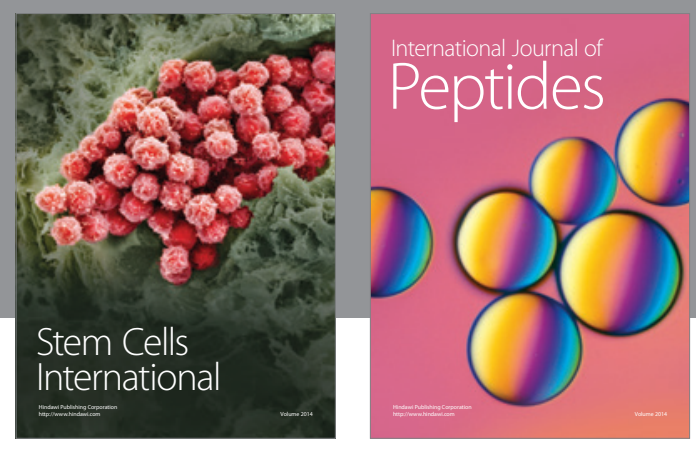

Submit your manuscripts at

http://www.hindawi.com
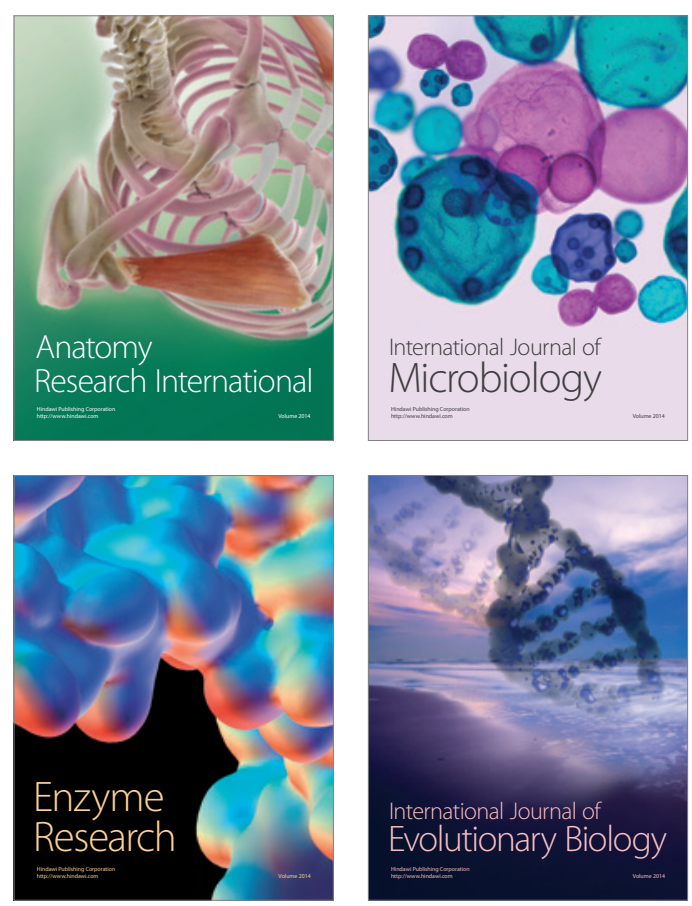
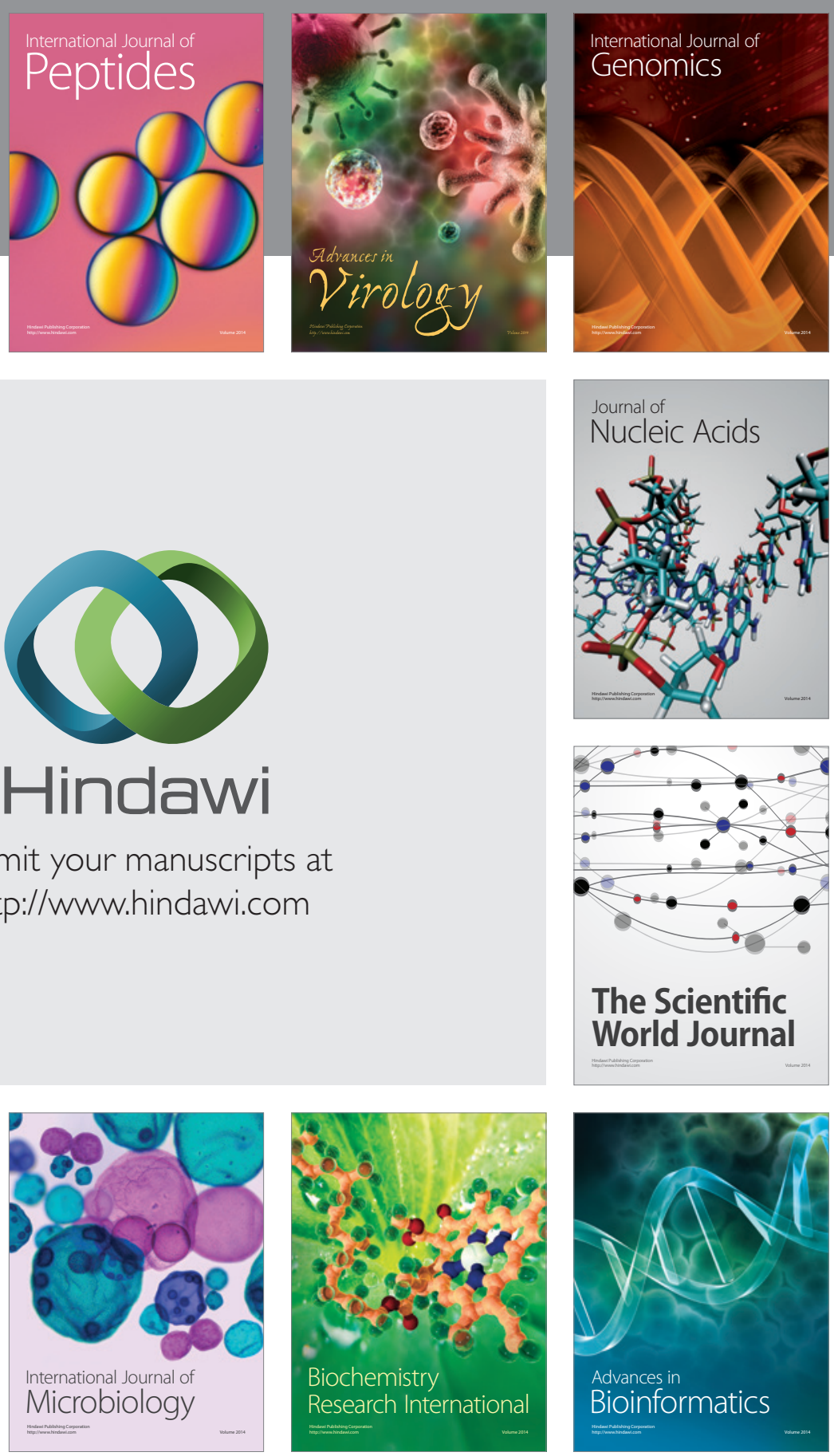

The Scientific World Journal
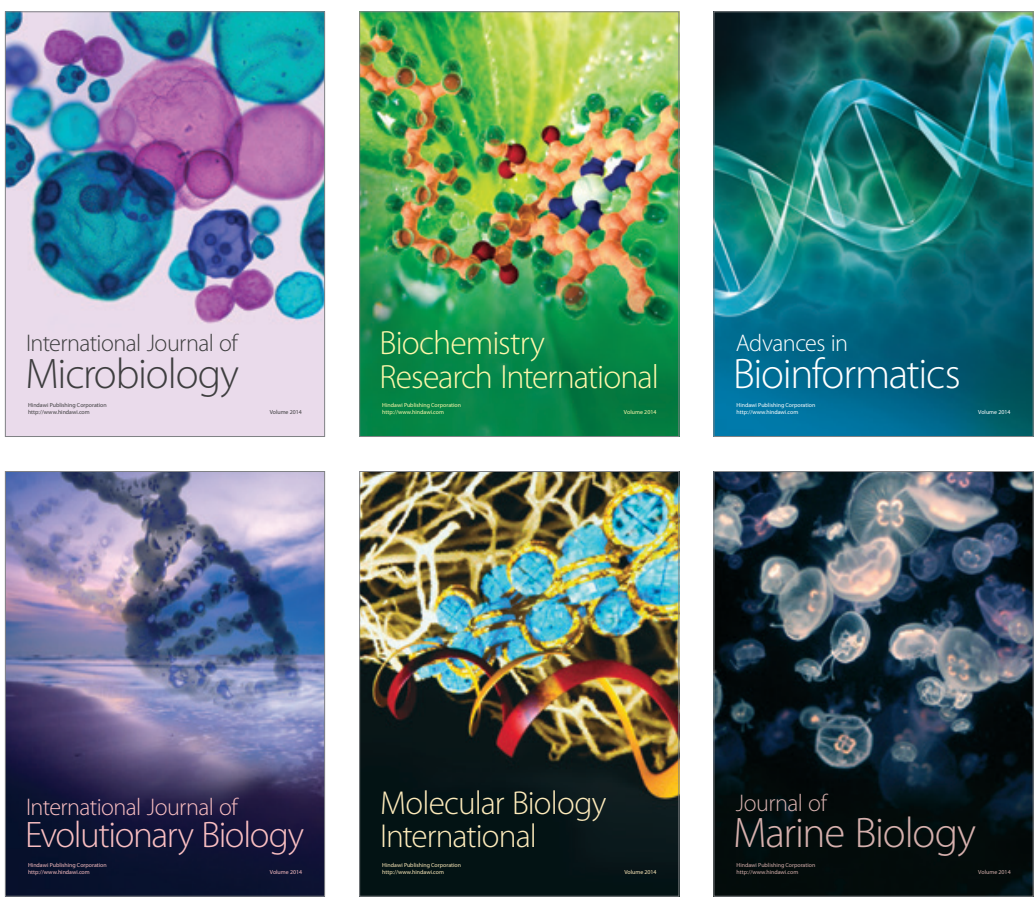